\title{
Modelling Directional Effects on Remotely Sensed Land Surface Temperature
}

Sofia L. Ermida ${ }^{1}$, Carlos C. DaCamara ${ }^{1}$, Isabel F. Trigo ${ }^{2}$, Ana C. Pires ${ }^{2}$, Darren Ghent ${ }^{3}$, and John Remedios ${ }^{3}$

1 Instituto Dom Luiz (IDL), University of Lisbon, Lisbon, Portugal

2 IPMA - Instituto Português do Mar e da Atmosfera, Department of Meteorology and Geophysics, Lisbon, Portugal

3 University of Leicester, Leicester, UK

\section{Corresponding Author:}

Sofia L. Ermida

snermida@fc.ul.pt

+351217500868

Faculdade de Ciências da Universidade de Lisboa, Campo Grande, Ed. C8, sala 8.3.15, 1749-016

Lisboa, Portugal

\section{ABSTRACT}

Land Surface Temperature (LST) is a markedly directional variable and its remotely sensed measurement may be strongly affected by viewing and illumination geometries. This study proposes the use of LST products collocated in space and time, but obtained with different viewing angles, to calibrate a simple model capable of characterizing the LST angular variability. The exercise is performed using MODIS (Aqua and Terra) and SEVIRI (Meteosat) LST products, for an area covering Mediterranean Europe and Northern Africa and encompassing the full years of 2011, 2012, 2013 and 2014. The approach relies on a kernel model that is composed by an "emissivity kernel" and a "solar kernel", associated to observation angle anisotropy and to shadowing/sunlit effects on the surface, respectively. The spatial distribution of the kernel coefficients is shown to reflect characteristics of the landscape, both in terms of vegetation cover and topography. Model performance is assessed through several comparison exercises over the 4-year period under analysis. Cross-validation results show that the angular correction by the kernel model leads to a decrease of the root mean square difference between SEVIRI and MODIS daytime (night-time) LST products, from the original uncorrected values of $3.5 \mathrm{~K}(1.5 \mathrm{~K})$ to $2.3 \mathrm{~K}(1.3 \mathrm{~K})$. Comparison of both MSG and MODIS LST products against in situ daytime measurements gathered over 2 years at a validation site in Évora (Portugal) reveals that the angular correction leads to a decrease in root mean square error from 4.6 $\mathrm{K}(2.0 \mathrm{~K})$ to $3.8 \mathrm{~K}(1.9 \mathrm{~K})$ for MODIS (SEVIRI). The kernel model may be a useful tool to quantify the LST uncertainties associated with viewing and illumination angles.

Keywords: Land Surface Temperature; directional effects; kernel model 


\section{INTRODUCTION}

Land surface temperature (LST) is defined as the radiometric skin temperature over land that results from the energy balance at the surface (Norman and Becker, 1995). As a component of the surface radiation budget playing a relevant role in the partition between sensible and latent heat fluxes, LST is an important parameter for the diagnosis of surface conditions (e.g. Xu et al., 2011; Wang et al., 2014). Remote sensing observations constitute the most effective means to monitor LST over large areas and on a regular basis. Most satellite LST products rely on measurements in the thermal infrared (IR) atmospheric window, i.e. within the 8-13 $\mu \mathrm{m}$ range (e.g. Li et al., 2013). This band is particularly appropriate as it presents relatively weak atmospheric attenuation under clear sky conditions and includes the peak of the Earth's spectral radiance (considering surface temperature of the order of $290 \mathrm{~K}$ that leads to maximum emission at about $10 \mu \mathrm{m}$, according to Wien's displacement law).

Since estimates of LST from infrared satellite observations correspond to the radiometric temperature of the surface as seen within the sensor field of view (FOV), remotely sensed LST is a directional variable (Norman and Becker, 1995). Given the high spatial heterogeneity of land surface, such directionality may lead to significant differences among LST products obtained for the same area and observation time, but with different viewing geometries (Lagouarde et al., 2000, 2004; Pinheiro et al., 2004, 2006; Barroso et al., 2005; Trigo et al., 2008; Ermida et al., 2014, Duffour et al., 2015). This effect contributes to enhance the differences among LST satellite products, and therefore increasing the challenge of using multi-sensor and multi-decadal data to provide harmonised LST datasets suitable for long-term climate observations. Accurate estimates of the angular effects on retrieved LST are also crucial when performing in situ and cross-sensor validation exercises (Ermida et al., 2014). Quantification of these effects may also be relevant when using LST for model assessment (e.g. Wang et al. 2014; Trigo et al., 2015) and data assimilation (e.g. English 2008; Ghent et al., 2010). 
The impact of the viewing geometry on LST estimations is related to a large extent with contrasts in the radiometric temperature of the various surface elements. In savannah-like landscapes, the measured difference between tree canopy and sunlit background during the dry season may reach over $20 \mathrm{~K}$ (Rasmussen et al., 2011; Ermida et al., 2014). Other factors such as orography or emissivity anisotropy may also play an important role (Trigo et al., 2008). The angular dependency of satellite observed LST should therefore be taken into account when combining LST products retrieved by different sensors, e.g., in order to obtain a harmonized variable.

There are several modelling studies of LST angular dependence (Minnis \& Khaiyer, 2000; Pinheiro et al., 2006; Rasmussen et al., 2010; Guillevic et al., 2013; Ermida et al., 2014; Duffour et al., 2015). However, the complexity of the processes associated to this dependence leads to physical models with a large number of time and space dependent parameters describing surface and vegetation properties, which makes their application difficult over large areas or long time-periods. Vinnikov et al. (2012) proposed a simple statistical model of angular anisotropy of LST that may be calibrated using collocated multi-sensor observations. The model follows a "kernel" approach commonly used for the description of the Bidirectional Reflectance Distribution function (BRDF) in the visible band (e.g. Jupp, 2000). The adequate characterization of the LST dependence on viewing and illumination angles could be useful to estimate the expected deviations of any given LST retrieval with respect to a reference view angle (e.g. nadir), and ultimately for the derivation of a directionally independent equivalent physical temperature, adequate to climate studies (Vinnikov et al., 2012).

Here we describe a feasibility study with the objective of assessing whether the kernel model proposed by Vinnikov et al. (2012) may be used to model the angular dependency of LST products using multi-sensor observations. For this purpose, the kernel model is applied to LST from sensors on board geostationary and polar-orbit platforms obtained over a large area covering Mediterranean Europe and over a multi-year time period. The model is evaluated for landscape-level thermal LST directionality, including vegetation and landcover-induced impacts as well as topographic effects. A 
description of the model is given in section 3, which also includes information on the LST products considered and the description of the model calibration/verification strategy. The model is then calibrated, verified and validated using LST data as obtained from the Spinning Enhanced Visible and Infrared Imager (SEVIRI) on-board Meteosat Second Generation (MSG) satellites (Trigo et al., 2011) and from the MODerate resolution Imaging Spectroradiometer (MODIS) on-board Aqua and Terra (Salomonson, Barnes, \& Masuoka, 2006). The model performance is also assessed by comparing both SEVIRI and MODIS LST products against in situ measurements of LST obtained at a validation site in Évora, Portugal. Results are discussed in section 4 and the main findings are summarized in section 5 .

\section{DATA}

Encompassing Mediterranean Europe, the study area is defined as the region between the latitude circles of $34.5^{\circ}$ and $45.8^{\circ} \mathrm{N}$ and between the meridians of $12.4^{\circ} \mathrm{W}$ and $30^{\circ} \mathrm{E}$ (Figure 1). The region is covered by very heterogeneous vegetation, ranging from dense to sparse forest, cultivated areas and shrubland (Figure 1).

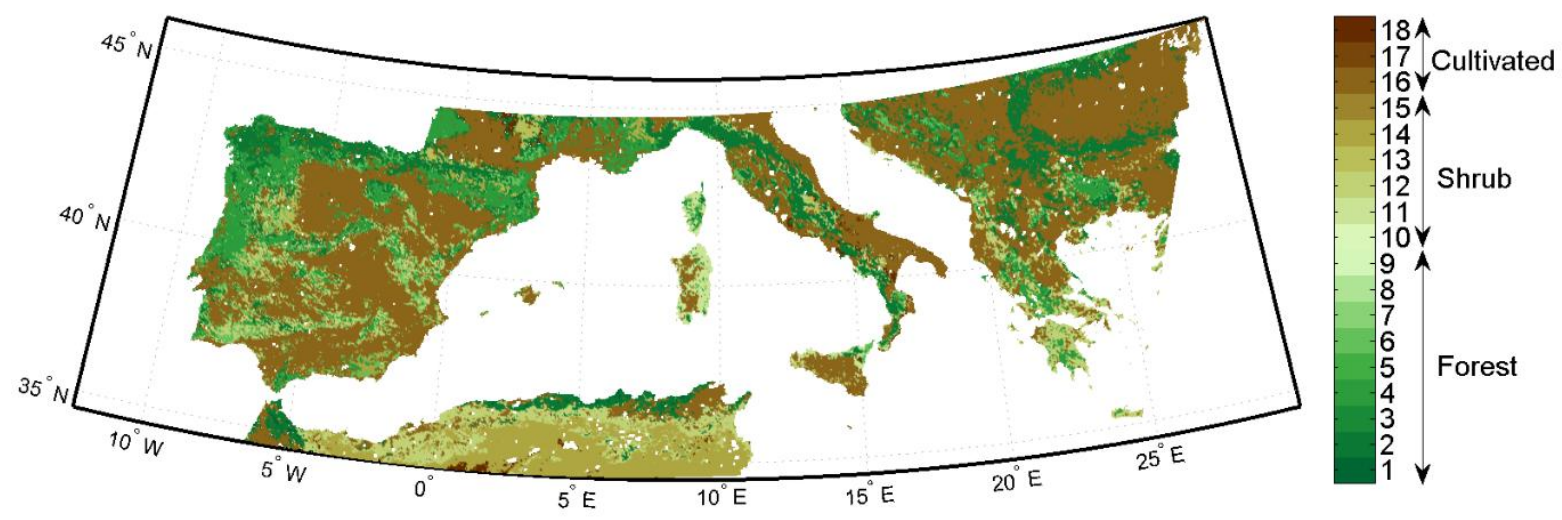

Figure 1 - Land cover classification according to GLC2000. Values of the colorbar correspond to GLC2000 labels. A detailed description may be found in http://bioval.jrc.ec.europa.eu/products/glc2000/legend.php. 


\subsection{SATELLITE LST DATA}

The kernel model was applied to satellite-observed LST obtained from two sensors: 1) the LST product retrieved from SEVIRI on-board MSG satellites provided by the EUMETSAT Satellite Application Facility on Land Surface Analysis (LSA-SAF) (Trigo et al., 2011); and 2) level 2 daily LST obtained from MODIS on-board Aqua (product MYD11, collection 5) and Terra (product MOD11, collection 5) (Wan, 2008). SEVIRI and MODIS LST for the whole area of study were obtained from the GlobTemperature portal (http://data.globtemperature.info/), which provides LST data in a standardized format, facilitating collocation in space and time and organization of needed auxiliary variables (including viewing geometry and sun angles).

The LSA-SAF LST is estimated with a generalized split-window algorithm (Freitas et al., 2010) from top-of-atmosphere brightness temperatures measured by MSG/SEVIRI in the thermal infrared, namely in SEVIRI channels IR10.8 and IR12.0. It is produced at full SEVIRI spatial and temporal resolutions, with a 15-minute sampling interval and a spatial resolution of $3 \mathrm{~km}$ at the sub-satellite point, which degrades with increasing distance from nadir, reaching a size of about $4 \mathrm{~km}$ over Southern Europe. The product is available for all land pixels within the Meteosat disk under clear sky conditions; the actual area coverage depends on product uncertainty (LST retrievals with uncertainty estimates above $4 \mathrm{~K}$ are masked out) and can reach view zenith angles up to $70^{\circ}$ (Freitas et al., 2010). According to Göttsche et al. (2016), comparisons with four LSA SAF dedicated stations resulted in a mean absolute bias of SEVIRI LST of $0.1 \mathrm{~K}$, with daytime and night-time biases up to $0.7 \mathrm{~K}$ (but with opposite signs). Land Surface Emissivity (LSE) used by the LSA SAF in the derivation of LST is estimated with the Vegetation Cover Method (Caselles and Sobrino, 1989; Peres and DaCamara, 2005; Trigo et al., 2008). The method allows the determination of effective LSE as the weighted average of the emissivities of the dominant bare ground and vegetation type within a scene, using daily values of Fraction of Vegetation Cover (FVC) to obtained the respective weights. Emissivity values for vegetation and soil types were obtained from the International Geosphere-Biosphere Program (IGBP) land cover classes (Belward, 1996; Peres and DaCamara, 2005; Trigo et al., 2008). 
The MODIS LST product is derived with a Split Window Algorithm (Wan and Dozier, 1996; Wan et al., 2002) applied to bands 31 and 32 with a formulation similar to that adapted by the LSA-SAF team for SEVIRI (Trigo et al., 2011). The product (MOD/MYD11) is available at a spatial resolution of $1 \mathrm{~km}$ with a maximum of four clear sky observations per day. Information on viewing geometry was obtained from MODIS products MOD03 and MYD03, collection 5 (http://modis.gsfc.nasa.gov/). Radiancebased validation of the collection 5 of MODIS LST level-2 products at 42 sites indicated that LST uncertainties are within $\pm 2 \mathrm{~K}$, being within $\pm 1 \mathrm{~K}$ in most cases (Wan, 2014). In the case of MODIS, LSE is estimated with a similar method to the SEVIRI but using (static) land cover types (Wan and Dozier, 1996).

The data were grouped into four datasets of geostationary (SEVIRI) and polar-orbiter (MODIS) based LST products collocated in space and time, according to the temporal coverage: the full years of 2011, 2012, 2013 and 2014 (referred hereafter as SEVMOD11, SEVMOD12, SEVMOD13 and SEVMOD14, respectively).

Space and time collocation was performed as follows:

1) MODIS LST were projected onto SEVIRI/MSG geostationary grid by attributing each MODIS pixel to the closest SEVIRI pixel center and averaging all MODIS pixels corresponding to each SEVIRI pixel. It is worth noting that this procedure takes into account the actual pixel sizes of SEVIRI and MODIS.

2) SEVIRI LST was then linearly interpolated to MODIS observation time using SEVIRI (15minute) observations immediately preceding and succeeding MODIS observation. This interpolation is only performed if both SEVIRI observations are cloud-free, otherwise a single observation is used if the time difference between SEVIRI and MODIS retrievals is below 7.5 minutes. 


\subsection{IN SITU DATA}

The LST products derived from SEVIRI and MODIS were also compared against ground observations gathered at the LSA-SAF validation site of Évora that is maintained by the Karlsruhe Institute of Technology (KIT). The site is located in Southern Portugal $\left(8.00^{\circ} \mathrm{W} ; 38.54^{\circ} \mathrm{N}\right)$ within an area characterized by Quercus woodland and an understory dominated by herbs and grasses. In situ measurements are collected every minute by three infrared radiometers (Heitronics KT-15.85 IIP), observing the sunlit background, a tree crown and the sky at $53^{\circ}$ zenith angle, which is used to estimate down-welling reflective components (Göttsche et al., 2013). The radiometers provide measurements of brightness temperatures within the 9.6-11.5 $\mu \mathrm{m}$ spectral interval, with an absolute accuracy of $0.3 \mathrm{~K}$ (Göttsche et al., 2013). The available time series covers two full years of 2010 and from October 2011 to September 2012. Values of in situ LST were estimated for nadir view from measurements of brightness temperature based on the up-scaling technique proposed by Ermida et al. (2014). The up-scaling method is based on weighted averages of brightness temperatures of tree canopy, and sunlit and shaded ground, taking into account the respective fractions within any given pair of viewing and illumination angles. Those fractions are estimated using a geometric model of the site described in Ermida et al. (2014), while emissivity is taken from the dataset described in Seemann et al. (2007). Values of emissivity range between 0.946 and 0.976, the lower values being observed during the summer. In contrast with the procedure followed in Ermida et al. (2014), the site emissivity and the emissivity values used in LST (MODIS or MSG) retrievals considered here were obtained from independent sources. Satellite LST data used for this comparison correspond to the pixel, at the original resolution of each sensor, with center closest to the station's location, and with temporal colocation matching the 1-minute sampling of the radiometers.

\subsection{COMPLEMENTARY DATA}

A hill shading algorithm was applied to DEM data as provided by NOAA's Global Land One-km Base Elevation Project (GLOBE) which are available at $1 \mathrm{~km}$ resolution 
(http://www.ngdc.noaa.gov/mgg/topo/globe.html). The hill shading algorithm allows the estimation of the fraction of shaded surface in mountainous areas (section 3.2) and, for that reason, allows the evaluation of the kernel model over these regions.

Information on percentage of tree cover was obtained from MODIS Vegetation Continuous Fields (MOD44B, collection 5) provided on a yearly basis with a $250 \mathrm{~m}$ resolution (Townshend et al., 2001). The data corresponds to the years of 2013 and 2014 and was re-projected onto the SEVIRI grid using plain average.

\section{Methodology}

\subsection{THE KERNEL MODEL}

The LST angular dependence is modeled through a kernel approach proposed by Vinnikov et al. (2012), and referred to hereafter as the kernel model. The kernel model provides the dependence of LST on viewing and illumination geometries by means of a statistical approach that is expressed by the following equation:

$$
\frac{T\left(\theta_{\mathrm{v}}, \theta_{\mathrm{i}}, \Delta \phi\right)}{T_{0}}=1+A \Phi\left(\theta_{\mathrm{v}}\right)+D \Psi\left(\theta_{\mathrm{v}}, \theta_{\mathrm{i}}, \Delta \phi\right)
$$

where $\left(\theta_{\mathrm{v}}, \theta_{\mathrm{i}}, \Delta \phi\right)$ are the view zenith, sun zenith and sun-view relative azimuth angles, respectively and $T_{0}=T\left(\theta_{\mathrm{v}}=0, \theta_{\mathrm{i}}\right)$ is the LST as viewed in the nadir direction. $A$ and $D$ are coefficients to be estimated from observations, that capture land cover structure. Following Vinnikov et al. (2012), the "emissivity kernel", $\Phi\left(\theta_{\mathrm{v}}\right)$, and the "solar kernel", $\Psi\left(\theta_{\mathrm{v}}, \theta_{\mathrm{i}}, \Delta \phi\right)$, are defined by the following expressions:

$$
\begin{gathered}
\Phi\left(\theta_{\mathrm{v}}\right)=1-\cos \left(\theta_{\mathrm{v}}\right) \\
\Psi\left(\theta_{\mathrm{v}}, \theta_{\mathrm{i}}, \Delta \phi\right)=\sin \left(\theta_{\mathrm{v}}\right) \cos \left(\theta_{\mathrm{i}}\right) \sin \left(\theta_{\mathrm{i}}\right) \cos \left(\theta_{\mathrm{i}}-\theta_{\mathrm{v}}\right) \cos (\Delta \phi)
\end{gathered}
$$


The "emissivity kernel" is associated only to variations in the observation zenith angle, whereas the "solar kernel" models the shadowing/sunlit effects on the surface, which lead to inhomogeneous heating due to the different thermal behavior of the various surface components (Vinnikov et al., 2012). As a first approximation for highly complex land surfaces, the emissivity kernel $\Phi$ is based on the assumption that emissivity only presents significant deviations from a nadir value for relatively high view zenith angles (larger than about $40^{\circ}$ ). Many surfaces do not show any significant variation at all. In the case of bare-ground surfaces, emissivity tends to decrease with view zenith angle, being otherwise isotropic (i.e. A 0) (e.g. García-Santos et al., 2012). Since the LST retrieval is performed using emissivity values that do not account for this variability, a decrease (increase) of emissivity with view angle will lead to LST values retrieved with emissivities that are too high (low), resulting in negative (positive) values of $A$. The shape of the solar kernel $\Psi$ models the impact of hot-spot and shaded surfaces within the FOV, with intensity depending on illumination angles and viewing geometry.

Since nadir observed LST data are quite uncommon, equation (1) must be rewritten to accommodate two LST time-series with different viewing configurations:

$$
\frac{T_{1}}{T_{2}}=\frac{1+A \Phi_{1}+D \Psi_{1}}{1+A \Phi_{2}+D \Psi_{2}}
$$

where $\Phi_{\mathrm{n}}=\Phi\left(\theta_{\mathrm{v}, \mathrm{n}}\right)$, and $\Psi_{\mathrm{n}}=\Psi\left(\theta_{\mathrm{v}, \mathrm{n}}, \theta_{\mathrm{i}, \mathrm{n}}, \Delta \phi_{\mathrm{n}}\right)$ and sub-index $\mathrm{n}=1,2$ denotes the two LST timeseries $T_{\mathrm{n}}$ considered. The sensors used in this study have different orbits that result in different spatial and temporal resolutions. Furthermore, there are intrinsic differences in sensor type and LST algorithms, including calibration, atmospheric and surface emissivity corrections, amongst others, that contribute to differences between the LST products that are not related to geometry. These differences may be taken into account by assuming a bias between the LST products derived from 
different sensors. As discussed in section 3.3, this bias is defined a priori, allowing equation (4) to be linearized and thus be adjusted by a simple linear regression.

\subsection{HILL SHADE}

The consistency of the obtained kernel model over mountainous regions was assessed by comparing values of parameter $D$ with the characteristics of illumination as estimated by an hill shading algorithm (Burrough and McDonnell, 1998) applied to a Digital Elevation Model (DEM). The algorithm provides an hypothetical percentage of illuminated surface, F, based on light source and relief information:

$$
\mathrm{F}=\left(\cos \theta_{i} \cos \theta_{s}\right)+\left(\sin \theta_{i} \sin \theta_{s} \cos \left(\phi_{i}-\phi_{s}\right)\right)
$$

where $\theta_{s}$ and $\phi_{s}$ are respectively the slope and the aspect of the mountain. For any given position of the sun, the algorithm allows estimating the fraction of shaded and sunlit parts of the mountain that should translate into the values of the solar kernel parameter, $D$.

\subsection{Calibration}

The calibration of the kernel model was performed through the following procedure:

1. First, we need to remove any systematic differences between the two datasets, i.e., any offset or multiplicative factors that explain a significant part of MODIS - SEVIRI LST discrepancies. This broad sense bias was calculated by simple linear regression $\left(L S T_{M O D}=\right.$ $\left.a * L S T_{S E V}+b\right)$ restricted to night-time observations and to similar viewing configurations by the two satellites $\left(\left|\theta_{\mathrm{v}, 1}-\theta_{\mathrm{v}, 2}\right| \leq 5^{\circ}\right)$. Such restrictions are aimed to exclude cases where sources of non-systematic MODIS - SEVIRI LST differences are most relevant. Over the study area and for the 2013-2014 period there are a total of 7,844,745 available matchups 
resulting in values of slope $a=0.981$ and intercept $b=4.71$. This bias correction was then applied to the SEVIRI LST product.

2. For each SEVIRI pixel within the study area, parameter $A$ was first calculated using MODIS and SEVIRI collocated LST (bias corrected) night-time observations (i.e., when $\Psi=0$ ):

$$
T_{1}-T_{2}=A\left(\Phi_{1} \mathrm{~T}_{2}-\Phi_{2} \mathrm{~T}_{1}\right)
$$

3. For each SEVIRI pixel within the study area, parameter $D$ was then calculated using daytime observations only and using values of $A$ obtained in the previous step:

$$
T_{1}-T_{2}-A\left(\Phi_{1} \mathrm{~T}_{2}-\Phi_{2} \mathrm{~T}_{1}\right)=D\left(\Psi_{1} \mathrm{~T}_{2}-\Psi_{2} \mathrm{~T}_{1}\right)
$$

To avoid taking into account LST values with very high uncertainties, only pixels with view zenith angles below $50^{\circ}$ were considered for the bias correction (step 1 in the list above). For the same reasoning, the estimation of A (step 2) used MODIS observations limited to view angles up to $50^{\circ}$. The kernel parameters, $A$ and $D$ (steps 2 and 3), are adjusted on a pixel-by-pixel basis, i.e., each considers a fixed SEVIRI viewing geometry. As such, the restrictions imposed to MODIS observations were relaxed for SEVIRI in order to maintain the area of study (see Figure 1), where SEVIRI view zenith angles vary from $39.75^{\circ}$ in the south-west to $59.5^{\circ}$ in the far north-east of the domain. It is also assumed non-temporally dynamic values of $A$ and $D$, although values are expected to change for cover types with a high seasonality. This calibration methodology was first applied to the time series given by combining SEVMOD13 and SEVMOD14 datasets. Obtained parameters of emissivity and solar kernels will be referred to hereafter as the reference model. For cross-validation purposes, the kernel model was also calibrated using SEVMOD13 (SEVMOD14) data only, and then verified through its application to SEVMOD14 (SEVMOD13) data. Validation with independent data was accomplished by applying the reference model to the SEVMOD11 and SEVMOD12 independent datasets. 


\section{RESULTS}

\subsection{MODEL PARAMETERS}

Figure 2 shows the fields of $A$ and $D$ parameters estimated over the study area for the reference model, which is calibrated with SEVMOD13 and SEVMOD14 combined datasets. It is clear that the kernel model captures vegetation and most notably orography patterns, which are known to have the most significant effects on directionality of LST (Trigo et al., 2008).
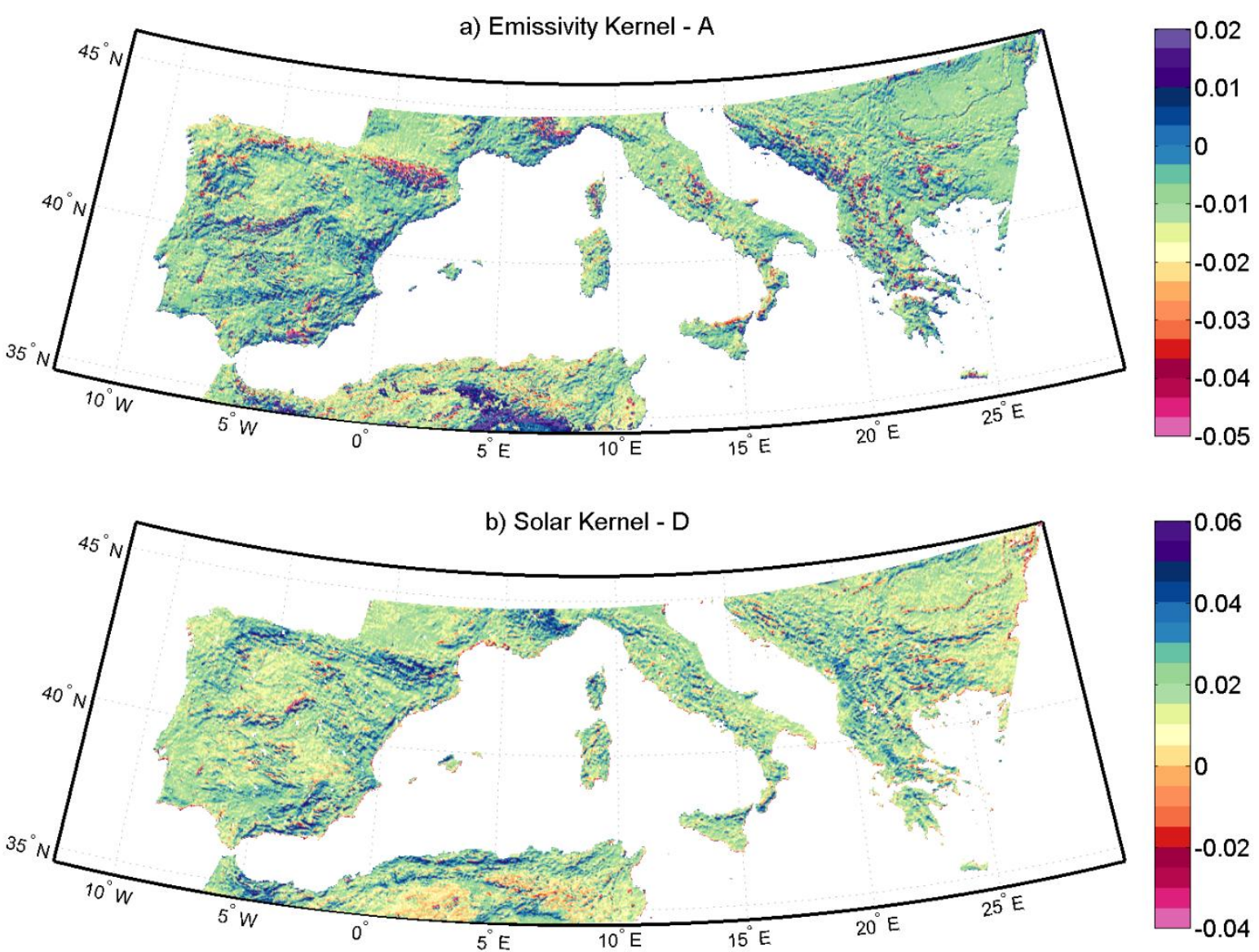

Figure 2 - Spatial distribution of coefficients of the reference kernel model for (a) A, the emissivity kernel (upper panel), and (b) D, the solar kernel. Colorbars are saturated at percentiles $0.2 \%$ and $99.0 \%$ for the emissivity kernel and at percentiles $0.5 \%$ and $99.7 \%$ for the solar kernel.

Fitting the emissivity kernel to the data leads mostly to negative values of parameter $A$ (Figure 2a), which indicates a decrease of the temperature relative to nadir for higher values of view zenith angle. According to the formulation in Vinnikov et al. (2012), this could be associated to a dependence of 
emissivity on view angle, where negative values of $A$ would be consistent with a decrease of emissivity with view angle. Emissivity is known to decrease with view zenith angle for homogenous surfaces like sea and bare ground but this variation is expected to be negligible for other surfaces like grassland (Labed and Stoll, 1991; Sobrino and Cuenca, 1999). Ren et al. (2011) assessed the pixel-scale directionality of emissivity and found that although emissivities in the thermal infrared band decrease with viewing angles, the angle dependency is very slight from $0^{\circ}$ to $45^{\circ}$ and only becomes significant for angles larger than $45^{\circ}$. Since only view angles up to $50^{\circ}$ are considered in the adjustment of $A$, it is possible that the adjusted values do not translate to emissivity angular dependence, but are rather a consequence of other factors that induce higher differences between the two LST products for large angles. Next we take a closer look at the distribution of parameter $A$ to further understand those factors.

Visual inspection of Figure 2a suggests a relation between values of parameter A and topography. Figure 3a shows the distribution of terrain topography (slope and orientation) per class of parameter $A$. It is shown that the highest absolute values of $A$ are found over mountainous regions, while flat surfaces are associated to $A$ values around -0.01 . Although one should be cautious when analyzing steep terrain, particularly because their geo-referencing errors tend to have a higher impact on LST uncertainties, Figure $2 \mathrm{a}$ and Figure $3 \mathrm{a}$ suggest a north-south gradient, with positive (negative) $A$ values over pronounced slopes facing south (north). This is consistent with the strong contrasting negative/positive bias between MODIS and SEVIRI LST over mountains such as Pyrenees or the Alps (Figure 4): the fixed SEVIRI viewing geometry roughly observing the area from south favors measurements of higher surface elevations over the northern part of mountain ranges, since lower valleys are more likely to be obscured/hidden within the field-of-view; this shading of low surfaces within SEVIRI FOV is highly attenuated in the case of southern slopes.

The spatial distribution of values of parameter A (Figure $2 \mathrm{~b}$ ) and of biases between MODIS and SEVIRI (Figure 4) suggest investigating the influence of the latter on the former. Figure $3 \mathrm{~b}$ presents 
the distribution of differences in MODIS and SEVIRI surface emissivities at $10.8 \mu \mathrm{m}$ per class of $A$. Regions where SEVIRI emissivity is higher than MODIS are associated to negative values of $A$, whereas regions with higher MODIS emissivity are associated to positive values of $A$. Figure $3 \mathrm{~b}$ suggests that $A$ tends to increase with increasing emissivity differences. The patch of very high $A$ values observed over the northern part of Africa corresponding to desert is particularly conspicuous in Figure $2 \mathrm{a}$, and is clearly associated to very high emissivity difference values between MODIS and SEVIRI over the same area (Figure 4); in Figure 3b, these correspond to the ridge in the $75 \%$ curve. Results from this analysis suggest that the adjustment of parameter $A$ in the kernel model acts as a further correction for local effects between MODIS and SEVIRI, which end up being superimposed on the emissivity angular dependency.
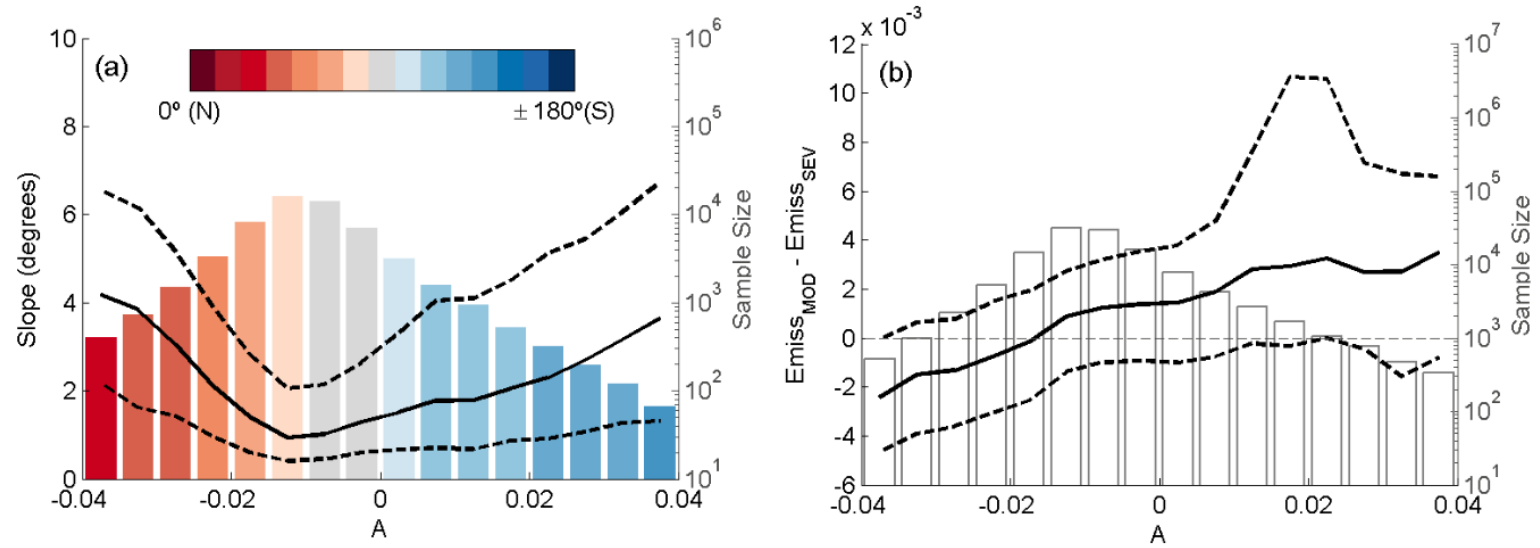
Figure 3 - Percentiles 50\% (solid line), 25\% and 75\% (dashed lines) of a) surface slope and b) of average $10.8 \mu \mathrm{m}$ emissivity differences between SEVIRI and MODIS over 2013 and 2014, for the classes of emissivity kernel parameter (A) indicated in the $x$-axis. The bars represent the sample size of each class (right $y$-axis). Colors of the bars in a) indicate the corresponding percentile 50\% of slope orientation (in degrees from North to South, in the eastward (positive) or westward (negative) directions).
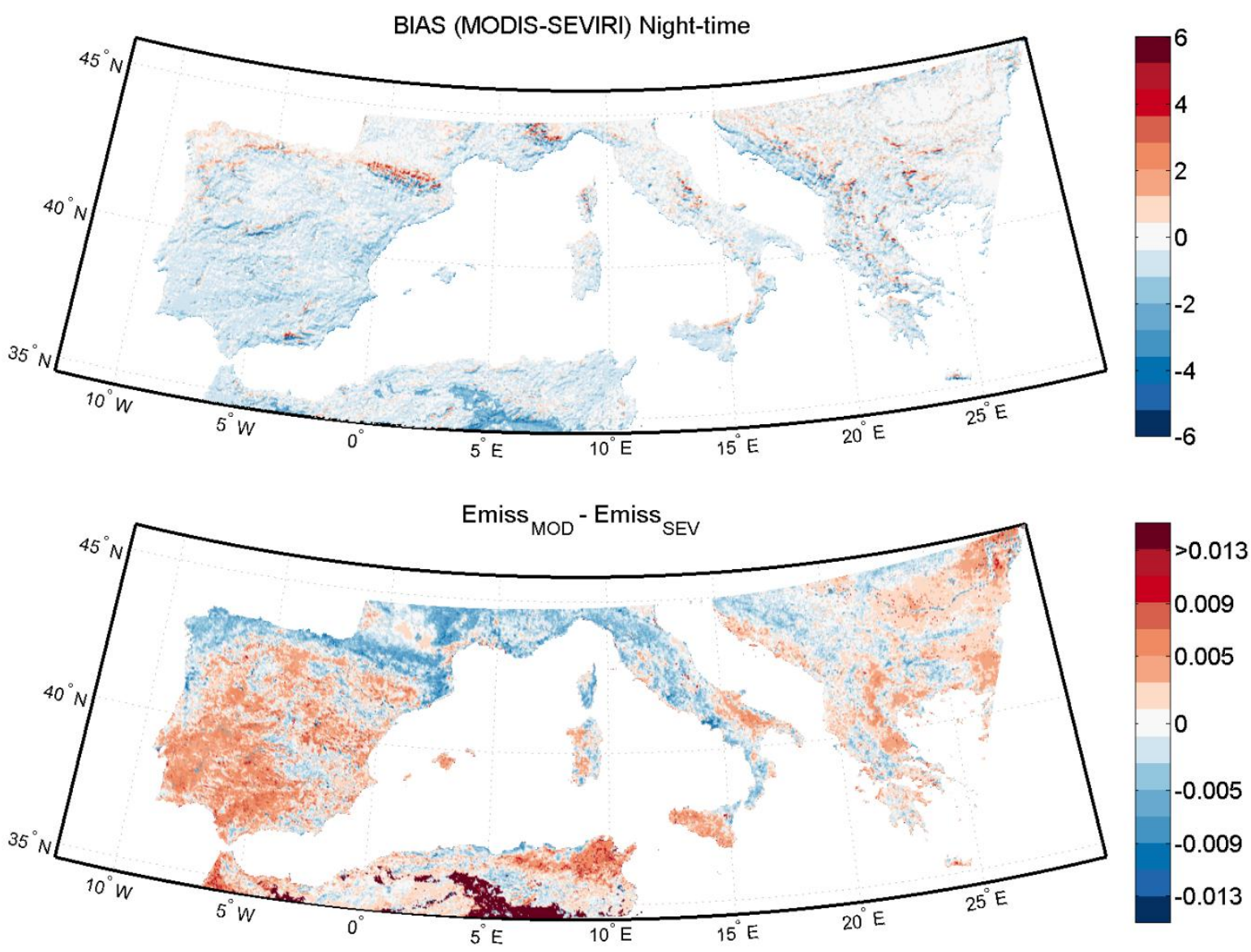

Figure 4-Spatial distribution of values of night-time bias between MODIS and SEVIRI LST (upper panel) and of the average differences between MODIS and SEVIRI emissivity of the $10.8 \mu \mathrm{m}$ channel over the years of 2013 and 2014 (lower panel).

Values of parameter $D$ (Figure $2 \mathrm{~b}$ ) as obtained by fitting the solar kernel to the data are mostly positive (only $6.4 \%$ are negative), indicating that LST increases relative to nadir in hot-spot situations (i.e. when the sun is effectively positioned behind the sensor) and decreases with view zenith angle for the remaining sun-sensor relative positions (as in the example shown in Figure 5). For heterogeneous pixels characterized by tree (or tall shrubs) coverage, the angular dependence of LST is mostly associated to shadowing effects. At the hot-spot the fraction of shadow observed by the sensor is significantly reduced as trees or other surface elements hide the shadow, leading the sensor to retrieve higher LST values. Off the hot-spot, the fraction of shadow observed by the sensor 
will increase with increasing view zenith angle resulting in lower LST values. This effect should be more relevant in areas with sparse or moderately vegetated surfaces as opposed to more homogeneous areas (bare ground or highly dense forests).
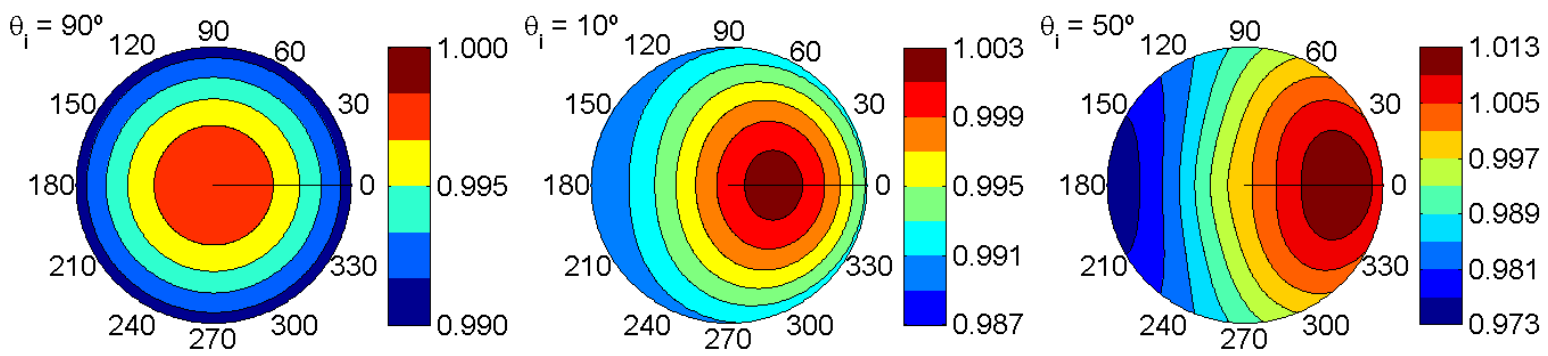

Figure 5 - Values of $T / T_{0}$ as given by the kernel model (eq. 1) for a common pair of $A$ and $D$ values $(A=-0.01$ and $D=0.04)$ and for three different values of solar zenith angle $\left(\theta_{i}\right)$ in a polar coordinate system: view zenith angle $\theta_{v}$ as radial coordinate $\left(0-90^{\circ}\right)$ and relative azimuth $\Delta \phi$ as angular coordinate $\left(0-360^{\circ}\right)$. Maximum of daytime $T / T_{0}$ is associated to the hot-spot effect - when the sun is effectively positioned behind the sensor.

The distribution of the percentage of tree cover (PTC) among several classes of $D$ values (Figure 6b) reflects the consistency of the spatial distribution of parameter $D$ with the patterns of vegetation (Figure 7). There is an increase of positive $D$ values with increasing tree cover. This is consistent with higher shadowing effects present in moderately dense forested areas. In contrast, values of $D$ closer to zero are dominated by low PTC values that correspond to less representative shadowing effects. Moreover, very dense forested areas may lead to a reduction in the angular dependence as the tree crowns obscure the shadows. As a result, the larger values of PTC are associated to intermediate values of $D$ (around 0.04).

More complex processes occur in mountainous areas, where LST retrievals depend not only on sensor-sun relative positions, but also on the relative orientation of slopes. There is an increase of D values with the surface slope (Figure 6a), which is consistent with the expected increased angular dependence. An illustration is provided of the case of the Guadarrama Mountains and the Pyrenes (Figure 8), two systems of mountain that range at the center and in the north of the Iberian Peninsula. The spatial distribution of parameter $D$ over the areas located in these mountain ranges tend to form bands of markedly positive and negative values (Figure $8 c, e$ ), which are in very close agreement with the fraction of illumination (Figure $8 \mathrm{~b}, \mathrm{~d}$ ) as provided by a hill shading algorithm (eq. 
5) applied to a DEM model of the area (estimated for a solar zenith angle of $20^{\circ}$, i.e., close to local noon). Despite the relatively noisy field of $\mathrm{D}$ values (Figure $8 \mathrm{c}$ and $8 \mathrm{f}$ ), higher positive (lower negative) values are associated to mountain slopes with higher (lower) fractions of illumination.
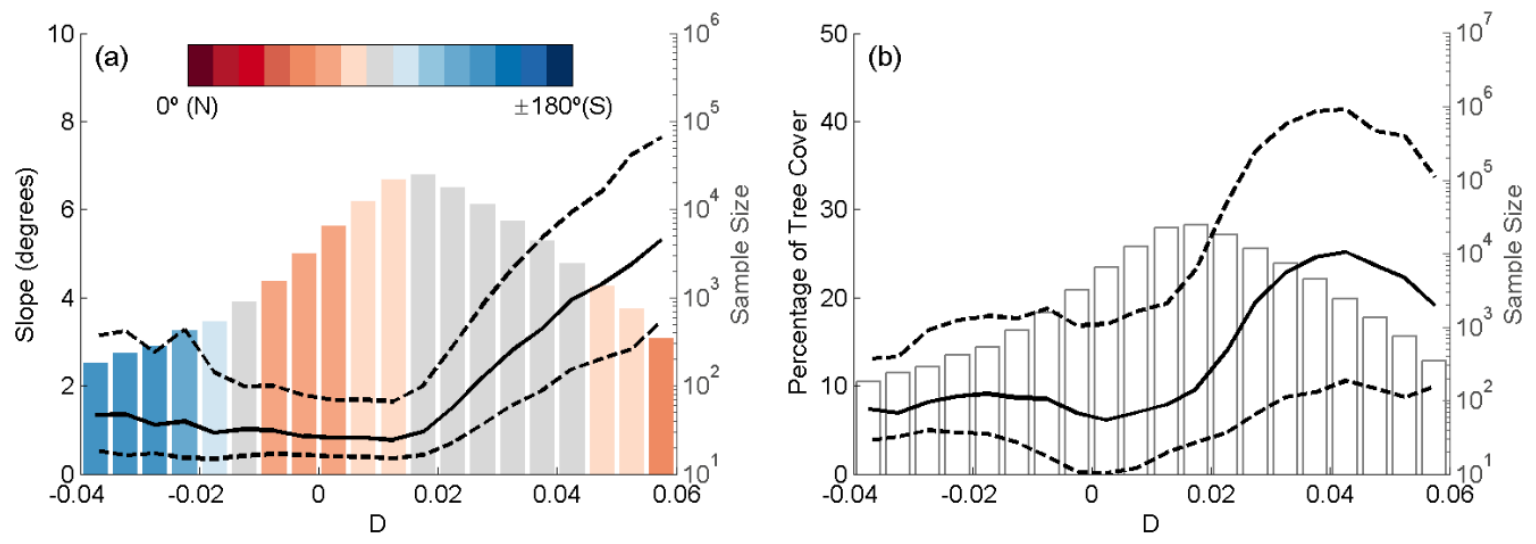

Figure 6 - Percentiles 50\% (solid line), 25\% and 75\% (dashed lines) of a) surface slope and b) Percentage of Tree Cover over 2013 and 2014, for the classes of solar kernel parameter $(D)$ indicated in the $x$-axis. The bars represent the sample size of each class (right y-axis). Colors of the bars in a) indicate the corresponding percentile 50\% of slope orientation (in degrees from North to South, in the eastward (positive) or westward (negative) directions).

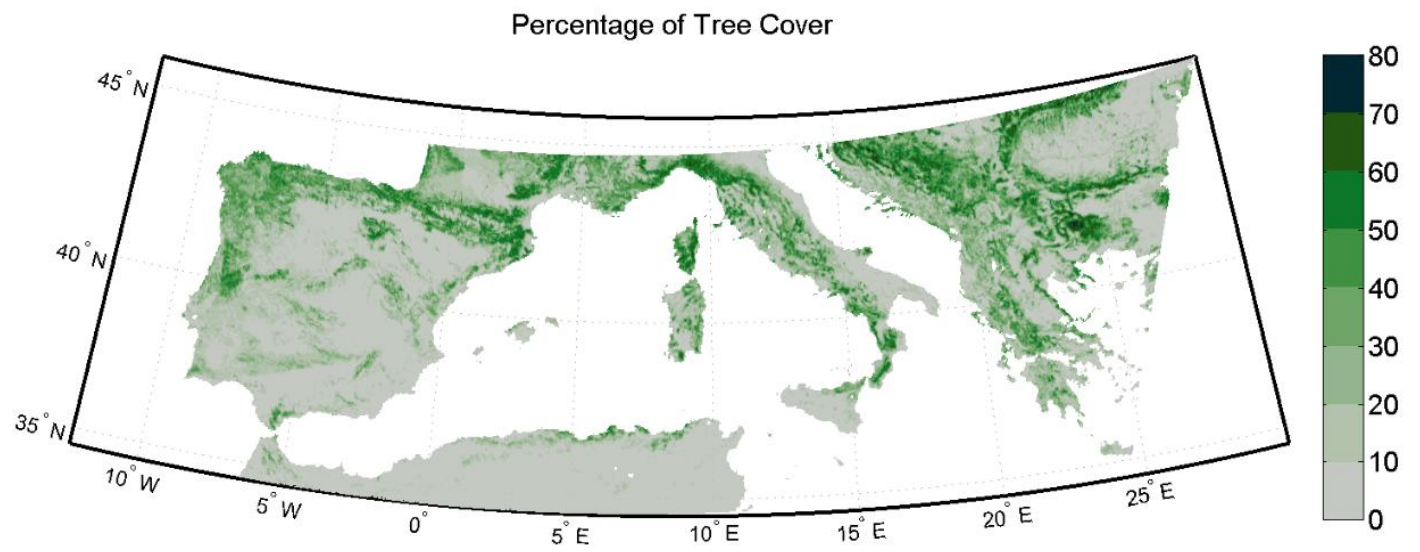

Figure 7-Percentage of tree cover as obtained from MODIS Yearly Vegetation Continuous Fields (product MOD44B) for the years of 2013 and 2014. 
a) Fraction of illumination $F$

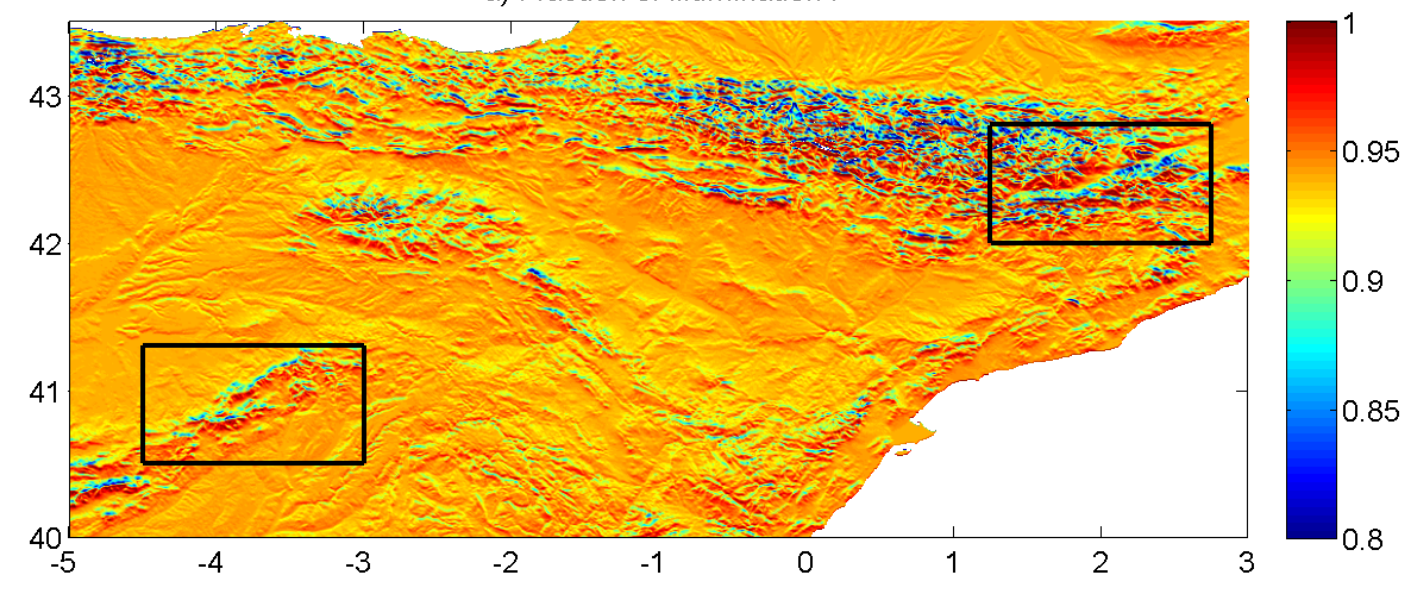

b) Guadarrama - Fraction of illumination $F$

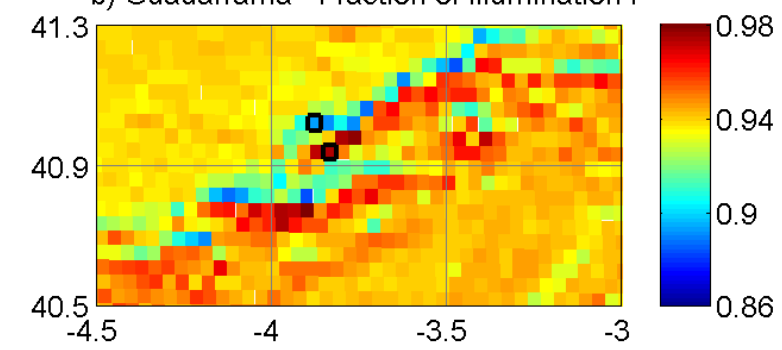

d) Pyrenes - Fraction of illumination $F$

c) Guadarrama - Solar Kernel parameter D
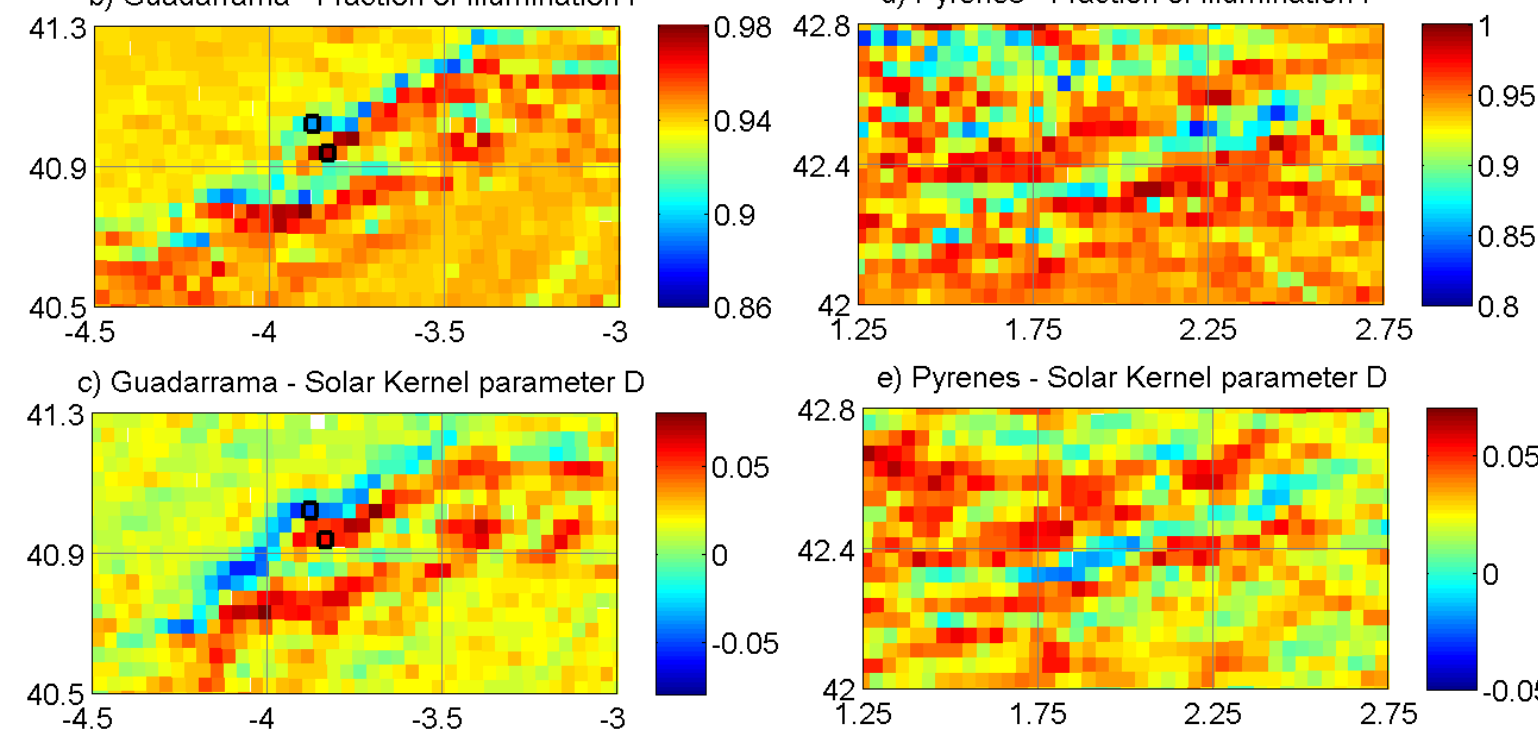

e) Pyrenes - Solar Kernel parameter D

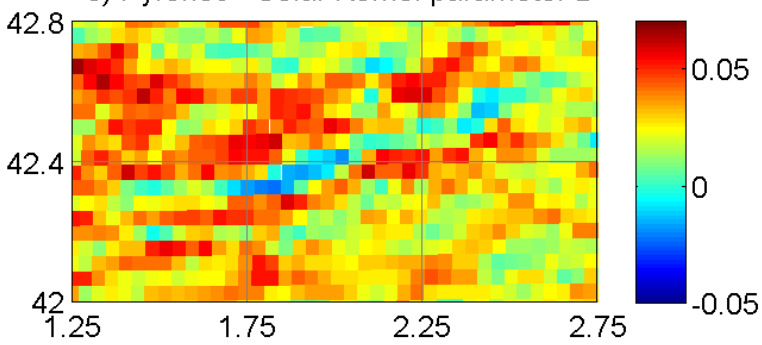

Figure $8-a)$ Fraction of illuminated surface, $F$, for sun zenith and azimuth angles of $20^{\circ}$ and $180^{\circ}$, respectively, as provided by a hill shading algorithm (eq. 5) applied to a $1 \mathrm{~km}$ DEM model of an area in northern Iberian Peninsula (rectangles denote the areas shown in panels $b$-e); $b, d)$ fraction of illumination, $F$, projected onto SEVIRI grid for the Guadarrama Mountains and the Pyrenes; and $c, d)$ the corresponding values of the solar kernel parameter $(D)$. The black squares in panels $b$ ) and $c)$ indicate the location of the SEVIRI pixels from which the LST time-series in Figure 9 were extracted.

An example of use of the developed kernel model is provided in Figure 9 that shows two LST diurnal cycles estimated from SEVIRI over two pixels located at the northern and the southern slopes of the Guadarrama Mountains, respectively. The different exposure of the hill slopes leads to opposite behaviors of LST dependence on view angle. For the northern pixel, a viewing geometry from south (like that of SEVIRI instrument) prevents the satellite from seeing the valleys that have in general higher temperatures than those at higher altitudes; therefore, an observation from nadir may result in higher LST values than one from south, as shown in Figure 9a. In the case of the southern pixel, the sunlit surface dominates, leading to higher values of temperature when the surface is observed 
from the south (Figure 9b). These results are also consistent with the fixed SEVIRI viewing geometry (from south), leading to a higher probability of low altitude (and therefore higher LST) to be obscured in northern slopes. Moreover, it is worth emphasizing that estimates of fraction of illumination (Figure 9a) were derived from a hill shading algorithm based on a DEM and are therefore independent from the estimates of the kernel parameters (Figure 9b) that were obtained from observed pairs of collocated values of LST by the SEVIRI and MODIS instruments.
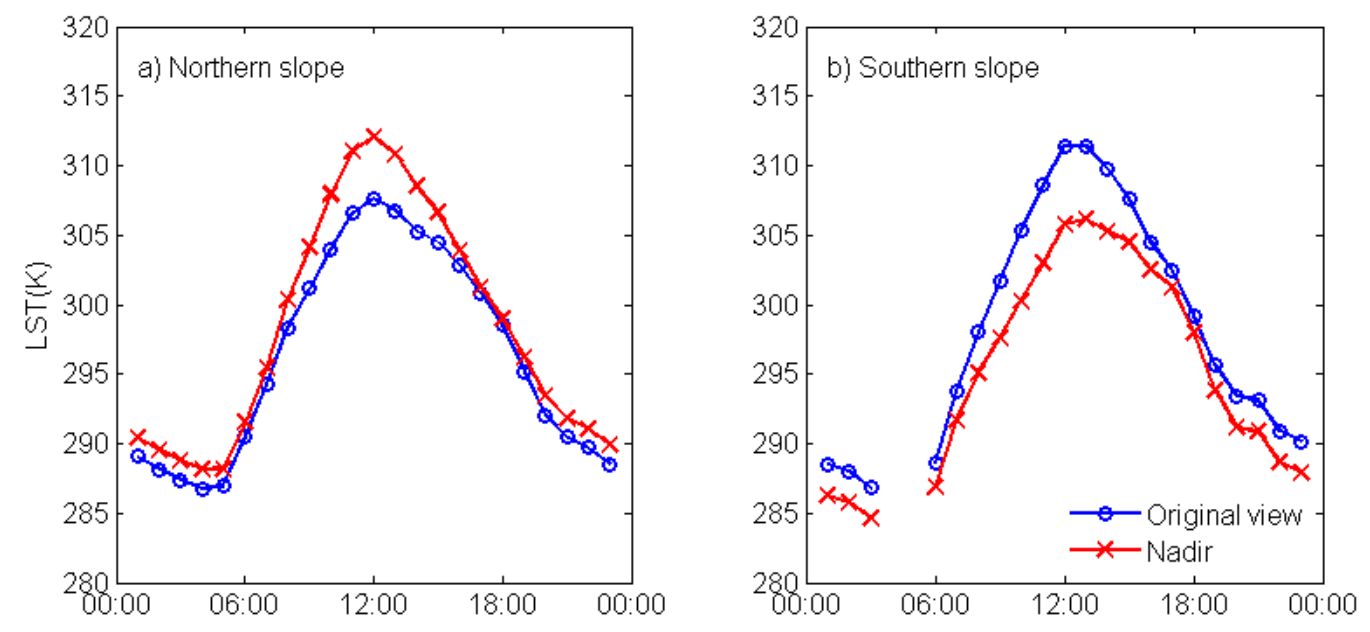

Figure 9 - Daily cycles of LST (K) as retrieved by SEVIRI (blue curves) and corrected to nadir view (red curves) on the $8^{\text {th }}$ of July of 2013 at the two points marked in Figure 8, respectively located a) on the northern slope and b) on the southern slope of the Guadarrama Mountains.

\subsection{VERIFICATION}

Performance of the model may be inferred by first carrying out an angular correction on one of the sensors' LST to the viewing configuration of the other and then comparing LST differences before and after the correction. The angular correction should lead to closer values of LST between the sensors. The reference model was used to correct SEVIRI LST to MODIS viewing geometry. Figure 10 (lower panel) presents the impact of angular correction, showing the change in the root mean square differences (RMSD) of LST values (i.e., [RMSD after angular correction] - [original RMSD]). There is an overall significant decrease in LST differences, up to $-3.6 \mathrm{~K}$, with a very small percentage of pixels (5.9\%) presenting an increase, but with lower amplitude. Larger RMSD decreases may be 
observed over mountainous areas as well as over a patch located in northern Algeria, where there are large differences in assigned values of emissivity by SEVIRI and MODIS.

Since the angular correction is performed on data that were bias corrected (calibration), the role of both corrections was assessed by means of a histogram of RMSD (Figure 11). It may be noted that each step leads to a shift of the distribution of LST RMSD towards lower values. The angular correction performed by the kernel model is nevertheless significantly more effective; the overall RMSD of $2.7 \mathrm{~K}$ decreases to $2.3 \mathrm{~K}$ after the bias correction, and to $1.8 \mathrm{~K}$ after the angular adjustment. As expected, daytime values present the most significant angular correction.
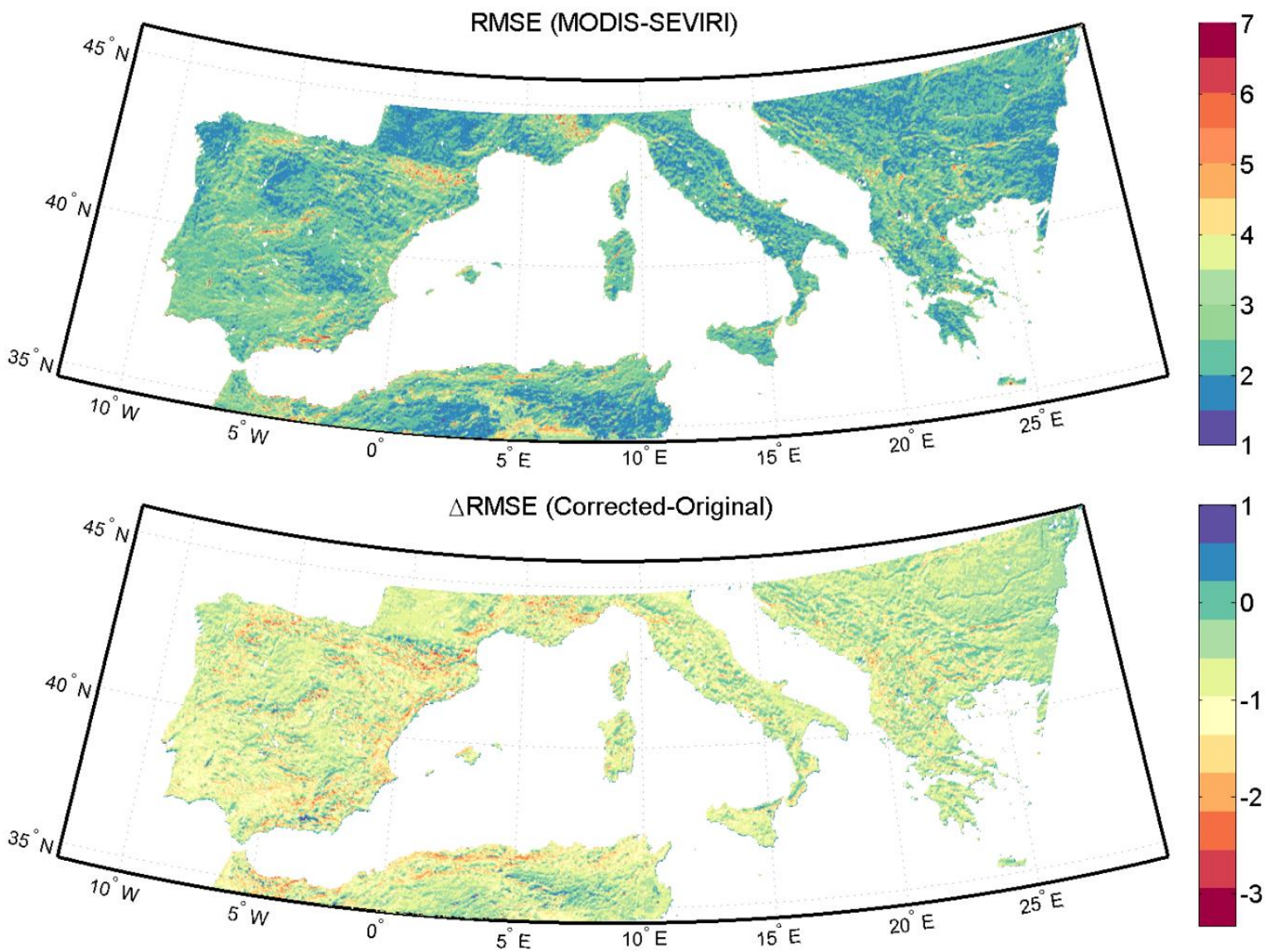

Figure 10 - Root Mean Square Differences (RMSD) between SEVIRI and MODIS (K) without angular correction (upper panel) and impact of LST angular correction on RMSD (in K) shown as RMSD after angular correction minus RMSD without correction (lower panel). 

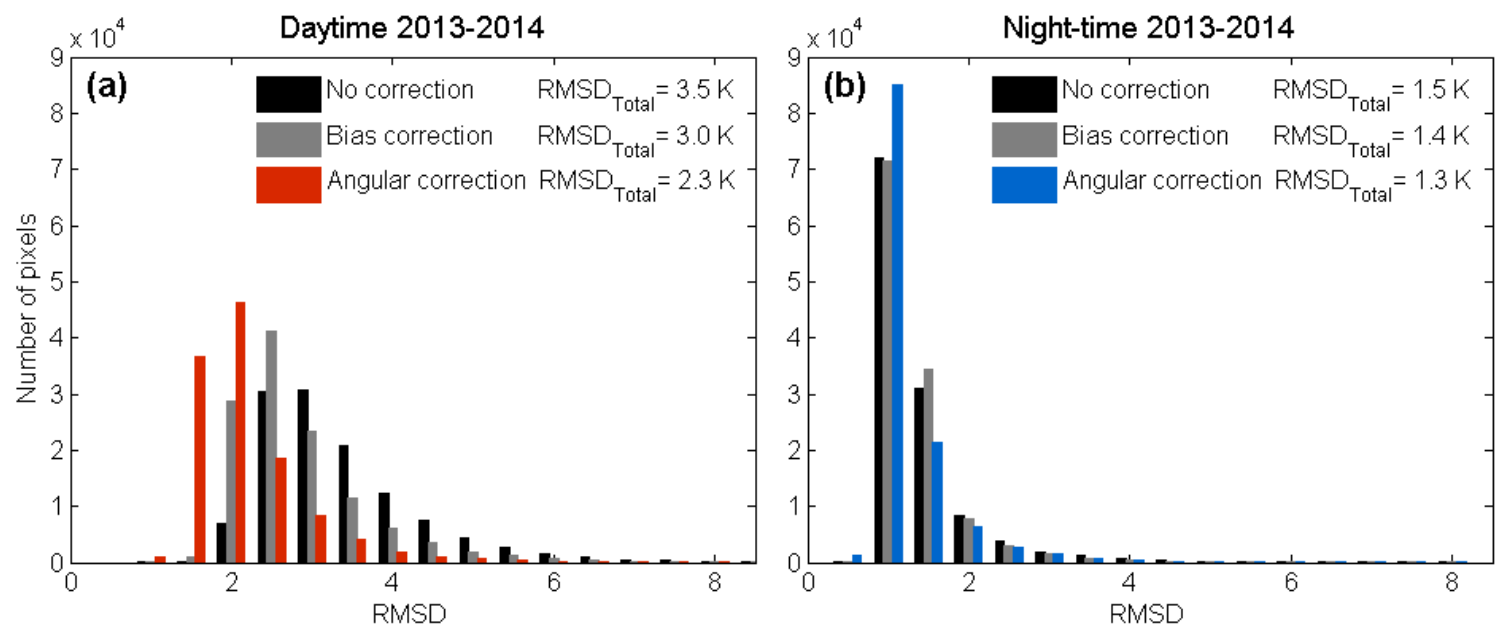

Figure 11 - Histograms of root mean square differences (RMSD) between SEVIRI and MODIS (in K) when no correction is applied (black), after applying the bias correction (grey) and after applying the angular correction with the reference model (red/blue) for (a) daytime and (b) night-time only observations.

A more detailed analysis reveals that only $3.7 \%$ of all pixels within the area of study present an RMSD degradation of $0.1 \mathrm{~K}$ or higher. These pixels, where discrepancies between SEVIRI and MODIS LST become larger after the angular correction, are associated to poor model fitting of equations (6) and (7) that lead to unlikely values of $A$ and $D$, namely: pixels where there is a RMSD degradation correspond to positive values of $A$ (51.6\% of the pixels) and/or to negative values of $D$ ( 77.5 of the pixels). As mentioned before, these pixels are mainly located in coastal and mountainous areas, where geo-referencing problems are likely to have a larger impact. In these cases, the quality or pixel representativeness of LST estimates may not be sufficient to perform a good calibration of the model.

\subsection{VALIDATION}

\subsubsection{Satellite-based}

The quality and robustness of the kernel model is assessed through two different processes: 1) a cross validation with SEVMOD13 and SEVMOD14 datasets (see section 2.2); and 2) an independent validation with the SEVMOD11 and SEVMOD12 datasets. 
For the cross validation, the kernel model is calibrated using SEVMOD13 and SEVMOD14 datasets separately, and then assessed through the application of those model parameters to SEVMOD14 and SEVMOD13, respectively. The aim of this procedure is to use independent datasets in the calibration and in the validation. The distribution of RMSD (Figure 12) is very similar to that obtained in the verification shown in Figure 11. It may be again noted that the angular correction presents the most pronounced impact on the retrievals, leading to the highest displacement of the histogram towards lower values. The performance of the kernel model seems therefore to hold, indicating that a kernel model calibrated with these data may be effectively applied to independent data.

Validation with independent data was performed by applying the reference model (i.e. calibrated with both SEVMOD13 and SEVMOD14) to SEVMOD11 and SEVMOD12. This procedure allows assessing the quality of the reference model when applied to a different time period.

The distribution of RMSD between MODIS and SEVIRI LST for the SEVMOD11 and SEVMOD12 datasets (Figure 12) presents characteristics very similar to those obtained before (Figure 11), with the highest impact being associated to daytime angular correction. These results together with those obtained in the verification and cross-validation processes strongly support the robustness of the kernel model. 

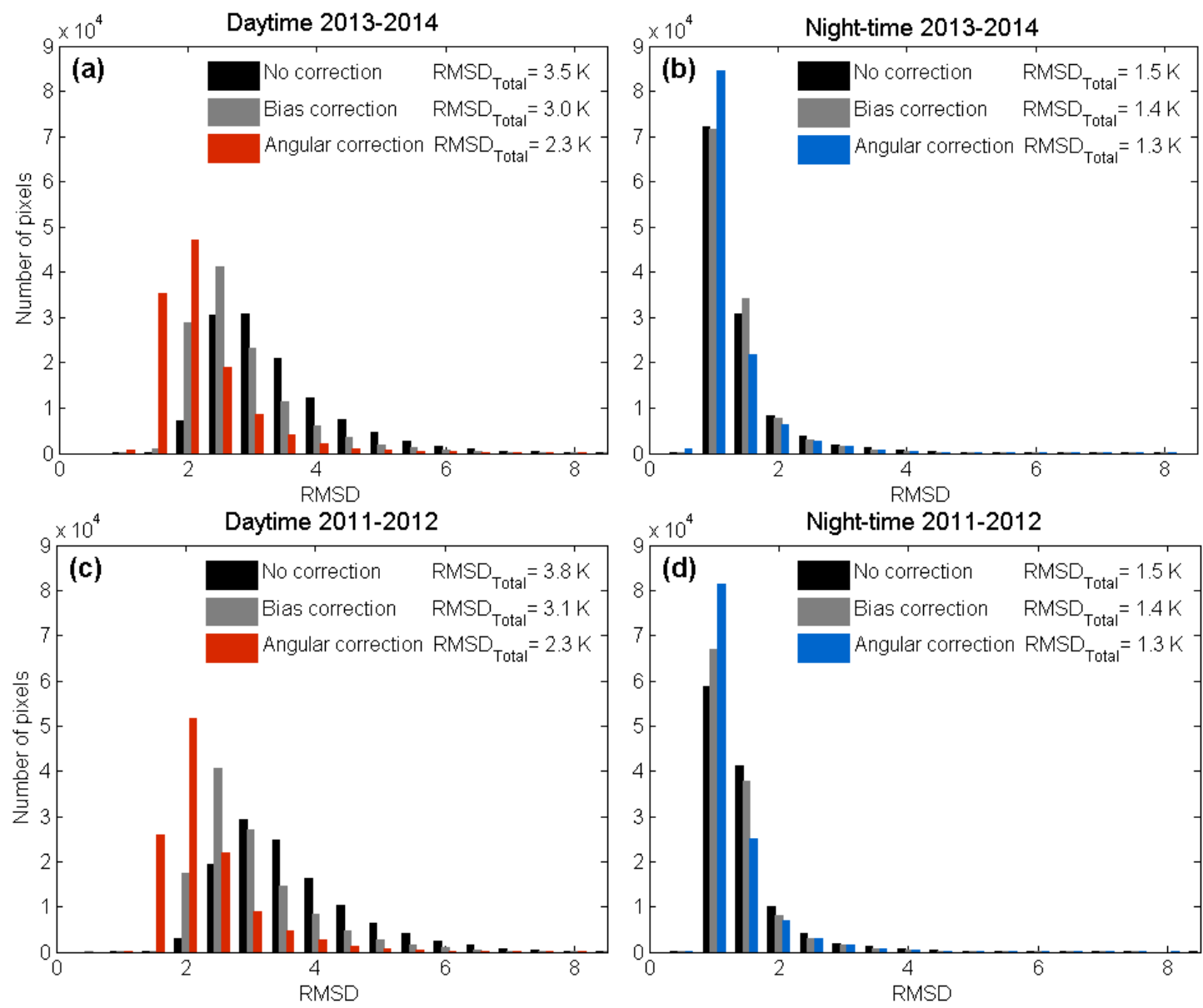

Figure 12- As in Figure 11, but $a, b)$ in cross-validation mode, i.e. results obtained with a model calibrated with SEVMOD13 data and verified with SEVMOD14 data together with a model calibrated with SEVMOD14 and applied to SEVMOD13 data and c,d) applying the reference model (calibrated with SEVMOD13 and SEVMOD14 datasets combined) to SEVMOD11 and SEVMOD12 data.

\subsubsection{In situ}

The Évora site (Portugal) maintained by KIT presents a unique set of observations for the validation of LST. As detailed in section 2.2, in situ observations combined with a geometric model of the landscape surrounding the station allow the up-scaling of ground measurements for any viewing geometry and for a wide range of illumination angles. As a first step, the ability of the kernel model to reproduce the essential features of the geometric model designed for Évora was assessed by comparing the distributions of T/TO ratio (where TO is the nadir LST) as a function of view zenith angle and relative azimuth valid for $\mathrm{T}$, as obtained by each model. In the case of the kernel model (Figure 13, left panel) values of T/T0 are those derived with the reference model at Évora. In the case of the geometric model (Figure 13, central panel), T/T0 field corresponds the median values along the year of in situ LST derived for different view and illumination geometry configurations. Although 
there are noticeable differences between the kernel derived from SEVIRI and MODIS time series and that derived from in situ observations, the main features are captured by the first one, namely the increase of T/TO towards nadir for geometries off the hot-spot zone. The ability of the kernel model to reproduce the patterns of the geometric model degrades as the solar zenith angle increases (not shown). This is to be expected since the kernel model does not take into account the lower differences in temperature among components that occur for higher solar zenith angles (namely, in winter and early morning and late afternoon). Indeed, while the sampling of the viewing geometry is restricted to collocated SEVIRI and MODIS observations in the case of the kernel model (Figure 13, right panel), the geometric space is entirely sampled in the case of the geometric model.
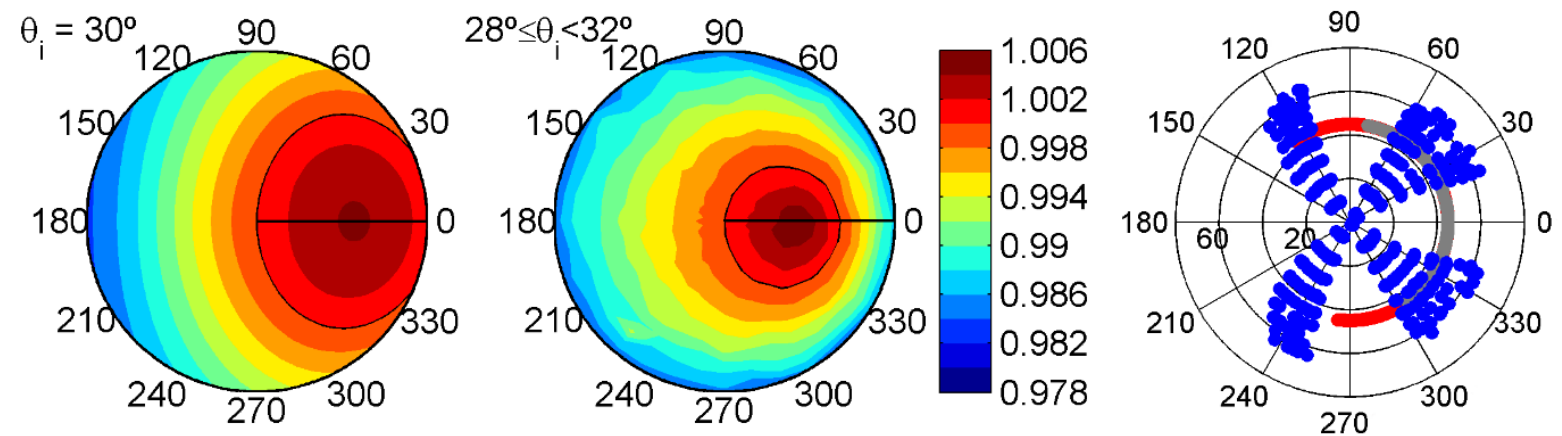

Figure 13 - As in Figure 5 but showing the values of $T / T_{0}$ as given by the reference kernel model at the Évora site (left panel) and as given by the geometric model (central panel), and showing the angle sampling of the LST time-series (right panel) of SEVIRI (red and grey dots), SEVIRI collocated with MODIS (grey dots) and MODIS (blue dots). The black lines in left and central panels corresponds to contour $T / T_{0}=1$.

Then, LST data retrieved by SEVIRI and MODIS (at their original resolution) for the nearest pixel to the Évora site were compared with respective in situ observations. The analysis covered the full year of 2010 and from October 2011 to September 2012 and the comparison exercise was performed before and after using the reference kernel model (calibrated with both SEVMOD13 and SEVMOD14) to correct satellite-observed LST to a nadir view. The model parameters at the site result in nighttime corrections that are much lower than the uncertainty of both LST products. For that reason, the analysis was restricted to daytime. 
For both SEVIRI and MODIS, the angular correction performed by the kernel model leads to LST values closer to the nadir in situ LST (Table 1). As expected, MODIS presents the largest reduction in the differences to in situ, given the larger VZA range of values of this sensor when compared with SEVIRI: SEVIRI VZA at the site is approximately $45^{\circ}$, whereas MODIS VZA varies between nadir and $65^{\circ}$ (Figure 13, right panel). It is worth noting that changes in bias associated to the angular correction increase (decrease) when considering the whole SEVIRI dataset (when restricting to MODIS overpasses). This contrasting behavior is related to a reduce sampling of SEVIRI relative azimuth angles (Figure 13, right panel).

Table 1 - Root mean square error (RMSE), error standard deviation (STD) and bias for the differences between satellite LST and in situ LST (K), before (italics) and after (bold) using the kernel model to correct the satellite LST to nadir view. The number of available pairs of MODIS/station and SEVIRI/station LST is also shown (N). The second row in SEVIRI statistics corresponds to values obtained when limiting SEVIRI observations to MODIS overpass.

\begin{tabular}{|c|c|c|c|c|c|c|c|c|c|c|c|c|c|c|}
\hline \multirow{3}{*}{2010} & \multicolumn{6}{|c|}{ VZA $\leq 45^{\circ}$} & \multirow[b]{2}{*}{$\mathrm{N}$} & \multicolumn{4}{|c|}{$\operatorname{VZA}>45^{\circ}$} & & & \\
\hline & \multicolumn{2}{|c|}{ BIAS } & \multicolumn{2}{|c|}{ STD } & \multicolumn{2}{|c|}{ RMSE } & & \multicolumn{2}{|c|}{ BIAS } & \multicolumn{2}{|c|}{ STD } & \multicolumn{2}{|c|}{ RMSE } & $\mathrm{N}$ \\
\hline & & & & & & & & & & & & & & \\
\hline SEYIPI & -0.8 & -0.4 & 2.1 & 2.0 & 2.3 & 2.1 & 2186 & & & & & & & \\
\hline SEVIRI & 0.8 & -0.1 & 1.9 & 1.8 & 2.1 & 1.8 & 133 & & & & & & & \\
\hline MODIS & -0.6 & -0.1 & 2.1 & 1.9 & 2.2 & 1.9 & 99 & -3.2 & -1.9 & 2.8 & 2.3 & 4.2 & 2.9 & 46 \\
\hline \multicolumn{15}{|c|}{ 2011-2012 } \\
\hline \multirow{2}{*}{ SEVIRI } & -0.5 & -0.3 & 1.9 & 1.8 & 2.0 & 1.8 & 5450 & & & & & & & \\
\hline & -0.7 & -1.4 & 2.1 & 1.7 & 2.2 & 2.2 & 220 & & & & & & & \\
\hline MODIS & -3.5 & -3.1 & 2.3 & 2.0 & 4.2 & 3.7 & 112 & -6.0 & -4.3 & 3.7 & 3.7 & 7.0 & 5.6 & 76 \\
\hline
\end{tabular}

The seasonal variability of RMSE, STD and bias for the differences between satellite LST and in situ LST was also analyzed (Table 2). As might be expected, the discrepancies between satellite and in situ LST are smaller during winter (DJF) and larger during summer (JJA), in agreement with the respective magnitudes of LST. Most statistical measures for both satellites present an improvement after correction by the kernel model. In the case of SEVIRI, the bias is positive in winter and negative in summer, a behavior that is consistent with results obtained by Ermida et al. (2014). In the case of MODIS, the STD decrease after correction is more pronounced than in SEVIRI, reflecting the variable character of the viewing geometry of MODIS. 
Table 2 - As in Table 1 but for inter-seasonal variability of RMSE, STD, and bias computed over the two periods of 2010 and 2011/2012. SEVIRI statistics corresponds to values obtained using all SEVIRI observations.

\begin{tabular}{|c|c|c|c|c|c|c|c|}
\hline & \multicolumn{2}{|c|}{ BIAS } & \multicolumn{2}{|c|}{ STD } & \multicolumn{2}{|c|}{ RMSE } & $\mathrm{N}$ \\
\hline & & & & & & & \\
\hline$D J F$ & 0.4 & 0.2 & 1.5 & 1.6 & 1.5 & 1.6 & 1515 \\
\hline MAM & -0.7 & -0.3 & 2.1 & 2.0 & 2.2 & 2.0 & 1647 \\
\hline$J J A$ & -1.5 & -0.8 & 1.7 & 1.8 & 2.3 & 2.0 & 3128 \\
\hline SON & 0.1 & 0.1 & 2.0 & 1.9 & 2.0 & 1.9 & 1346 \\
\hline \multicolumn{8}{|l|}{ MODIS } \\
\hline$D J F$ & -2.4 & -1.3 & 1.7 & 1.4 & 2.9 & 1.9 & 80 \\
\hline MAM & -2.6 & -1.8 & 3.2 & 2.8 & 4.1 & 3.3 & 63 \\
\hline$J J A$ & -4.1 & -3.4 & 4.0 & 3.5 & 5.8 & 4.9 & 131 \\
\hline SON & -2.6 & -1.8 & 2.9 & 2.4 & 3.9 & 3.0 & 59 \\
\hline
\end{tabular}

\section{DISCUSSION}

The adjustment of the kernel model using SEVIRI and MODIS LST estimates collocated in space and time led to emissivity and solar kernels parameters, A and D, close to the values determined by Vinnikov et al. (2012). The values estimated for parameter $A$ are mostly negative suggesting that emissivity anisotropy contributes to a decrease of LST relative to nadir with increasing view zenith angle. However, systematic differences between the LST products at the pixel level (local biases) also contribute to the obtained values of $A$. Results show a strong dependence of values of $A$ on emissivity differences and surface orography (slope). Furthermore, in the case of hilly areas, values of A present contrasting north/south spatial patterns that closely follow the bias between MODIS and SEVIRI LST. This pattern in the bias might be related to the view angle of SEVIRI: SEVIRI observes the study area from south, which could result in SEVIRI being unable to observe the valley areas (where temperature values tend to be higher) of the northern slopes of mountains; as a consequence, SEVIRI LST tends to be cooler than MODIS on the northern side of mountains.

Parameter $D$ presents mostly positive values indicating that solar effects contribute to a decrease of the LST when viewing angles deviated from nadir, with the exception of the hot-spot effect (i.e., for identical viewing and illumination geometries). This is in accordance with the geometric considerations that indicate that the fraction of shadow seen by the sensor increases with view 
angle (Ermida et al., 2014). Only a reduced number of pixels present $D$ values that do not follow this behavior (6.4\%). These pixels either lie along the coastline, where geo-referencing problems have a high impact due to the contrasting temperature of water, or are located in mountainous regions. The high heterogeneity of the latter also enhances geo-referencing errors. In both cases, the collocated LST products correspond to mixed pixels (land-sea or multiple altitudes) and small mismatches in geo-location between different time-slots may result in poorer quality model fitting.

The kernel model originally proposed by Vinnikov et al. (2012) did not take into account shading geometry associated with sloping terrain. However, and despite the caveats referred above for those surfaces, the model is able to simulate LST anisotropy associated with heterogeneities induced either by vegetation or local orography. Improvement of the model by explicitly including these features may help increase quality of the regressions, leading to better quality of parameters over these regions. It is also worth noticing that vegetation cover and orography are generally correlated, which might make the partitioning between vegetation and topographic effects. Nevertheless, the obtained $D$ parameters seem to be consistent with the expected slope shading (Figure 8), which suggests that the solar kernel is able to statistically depict these features from the LST data.

Seasonal variations of the vegetation may impact the values of solar kernel $D$ as the shaded fraction of the pixel depends on vegetation cover. To allow for a higher number of SEVIRI-MODIS matchups to be available, this seasonal component was not taken into account. It may, nevertheless, be easily included by adjusting monthly (or even 3-monthly) parameters, given that an adequate amount of data is available.

Since there is no method available that would allow the validation of the model over the whole region of study, LST differences between the sensors were used as a measure of quality and consistency of the model. Results show that this correction leads to a significant decrease of LST differences between MODIS and SEVIRI, particularly during daytime when LST anisotropy is significant due to shadowing effects. This indicates that the model is effective and is able to reduce a 
large part of LST differences associated to LST angular dependence. The cross-validation and evaluation with independent data show that LST differences present a decrease that is consistent with the previous results, indicating that the model is robust. Robustness is very import in this case since it will allow for the model to be used operationally without the need of frequent recalibration, although the model parameters should be checked on a regular basis since changes in land cover may require recalibration.

The kernel model was shown to reproduce the essential features of the distribution of T/T0 values as derived from an in situ based geometric model, that provides a detailed description of the angular dependence of LST over Évora site. Differences between T/TO generated by the kernel and geometric models are apparent especially for high solar zenith angles. However, the kernel model cannot be expected to reproduce exactly the geometric model, which was designed for that specific site. While the former depends on two parameters that are statistically adjusted using two timeseries of collocated observations covering a limited portion of the viewing-illumination geometric space, the latter requires 7-to-8 local parameters (tree density and shape parameters, temperatures of different scene components and emissivity). A comparison of results from the kernel model with those from a geometric model at a single location have therefore to be analyzed with due care, taking into consideration 1) the complexity of the geometric model, 2) the limited geometries covered by collocated SEVIRI and MODIS data, and 3) the overall large-scale domain where the kernel model is to be applied.

The model performance was also assessed through comparisons with in situ LST data and discriminating satellite VZA below and above $45^{\circ}$ ( $45^{\circ}$ corresponds roughly to SEVIRI VZA over Évora). It should be noticed that in this case we compare swath (level 2) LST data from both MODIS and SEVIRI with in situ nadir view observations, taken here to be the reference view. For the year of 2010 and for VZA values below $45^{\circ}$, MODIS and SEVIRI products present similar RMSE values. The same is not true for the 2011-2012 dataset, where MODIS presents a RMSE higher than SEVIRI (Table 1). 
During 2012, the Iberian Peninsula was affected by a strong drought (Trigo et al. 2013) that led to a strong decrease in green vegetation cover, particularly in late spring/summer months. In contrast with SEVIRI, MODIS emissivity does not show the signature of such strong vegetation anomaly for 2012 in the region surrounding Évora station, resulting in the higher discrepancies (underestimation) with in situ observations. Values of emissivity at 10.8 micrometer from both Seemann et al. (2007) and from SEVIRI present a seasonal cycle and a marked decrease from 2010 to 2011/2012. The former range from 0.946 to 0.968 (0.957 and 0.976) from summer to winter in 2011/2012 (2010) and the latter from 0.969 to 0.977 (0.973 to 0.985$)$. In the case of MODIS, both the seasonal cycle and the changes from 2010 to 2011/2012 are very weak, the values of emissivity ranging between 0.982 and 0.984 for both time periods. Nevertheless, the impact of the kernel model angular corrections is positive for both sensors despite being limited to values within the products uncertainty (improvement in RMSE of the order of 0.2 to $0.3 \mathrm{~K}$; Table 1). As expected, MODIS RMSE is higher for higher VZA values and it is in this case that the kernel model shows higher corrections, with RMSE reductions of 1.3 to $1.4 \mathrm{~K}$ (Table 1 ). For angles above $45^{\circ}$ (MODIS retrievals only), the kernel model seems to be more efficient in reducing the bias with respect to in situ observations than the standard deviation of the differences (positive impact in 2010 and neutral in 2011-2012; Table 1).

\section{CONCLUDING REMARKS}

The current availability of long-term time series of physical and biophysical parameters derived from remotely sensed observations has opened new perspectives in monitoring Earth's surface. It is possible to estimate a wide range of land surface variables, including Land Surface Temperature (LST) data from many different sensors on-board geostationary or polar orbit platforms. The use of various algorithms, often based on very different assumptions, together with the diverse sensor spatial and temporal samplings and viewing perspective, makes it difficult to harmonize the various 
satellite products available (e.g., Barroso et al., 2005; Pinheiro et al., 2006; Rasmussen et al. 2010). This is particularly true in the case of LST, a markedly directional variable that, among other factors, is strongly affected by differences on viewing and illumination geometry.

The present study addresses the problem of modelling directional effects of LST products using time series of observations collocated in space and time from SEVIRI on-board Meteosat Second Generation satellites, and MODIS on-board Aqua and Terra. The approach relies on the kernel model proposed by Vinnikov et al. (2012) that is composed of an "emissivity kernel" and a "solar kernel". For each pixel, the kernel model is statistically fitted to unbiased pairs of SEVIRI and MODIS observations using a linear regression. The study area encompasses Mediterranean Europe and North Africa and the period analyzed covers the full years of 2011, 2012, 2013 and 2014.

The spatial distributions of parameters $A$ and $D$, the coefficients of the emissivity and solar kernels, respectively, reflect the characteristics of the landscape, both in terms of vegetation cover and topography. Values of A are mainly influenced by local biases between MODIS and SEVIRI LST products, which in turn are associated to a wide range of factors (e.g. emissivity differences, biases induced by surface heterogeneity and SEVIRI fixed viewing geometry). Those factors overlap the impact of emissivity angular dependence, which only has a relevant impact on LST for large view zenith angles. The higher values of parameter $D$ (solar kernel) are associated to areas dominated by moderately dense forest and indicate a strong dependence of LST on shadowing effects. Over mountainous regions, there is a close agreement between the spatial distribution of $D$ and that of the fraction of illumination. Both quantities tend to form stripes on the images corresponding to valeys, those with higher (lower) values of $D$ being associated to higher (lower) values of fraction of illumination.

The performance of the kernel model was assessed through several comparison exercises over the 3-year period under analysis, where the differences between collocated LST values obtained from MODIS and SEVRI/Meteosat are considered before and after the angular effects are taken into 
account. It is shown that the use of the kernel model to describe the angular variability of LST effectively reduces the differences between the two products. A cross-validation of the kernel model revealed that the root mean square difference (RMSD) between SEVIRI and MODIS daytime (night-time) products changed from the original values of $3.5 \mathrm{~K}(1.5 \mathrm{~K})$ to $2.3 \mathrm{~K}(1.3 \mathrm{~K})$, once the correction was considered. The larger decreases in local RMSD were observed over mountainous regions and over an arid patch over northern Algeria, reflecting the roles played by the solar and the emissivity kernels, respectively.

The daytime SEVIRI and MODIS LST were also compared against in situ observations taken from Évora (Southern Portugal, see Trigo et al, 2008; Ermida et al., 2014). The pixels surrounding the site present relatively low values for the kernel parameters, particularly in the case of the emissivity kernel. For this reason, only daytime observations, when directional effects are more pronounced, were considered. In this case, the angular correction led to an overall decrease in RMSE from $4.6 \mathrm{~K}$ (2.0 K) to $3.8 \mathrm{~K}(1.9 \mathrm{~K})$ for MODIS (SEVIRI), for the whole period under analysis.

Results of this study demonstrate that it is possible to use LST estimates collocated in space and time, but obtained with different viewing geometries, to calibrate a simple model capable of characterizing the LST angular variability. Here, the exercise was performed using MODIS (Aqua and Terra) and SEVIRI/Meteosat LST products, for an area covering Europe and Northern Africa. It has been shown that the model calibration is stable and it can be easily extended to other areas. As such, the kernel model may be viewed as a tool to estimate the expected LST uncertainty associated with viewing and illumination angles. But one should be aware that this model has several caveats and in particular Duffour et al (2015) have shown its limitation in simulating the correct amplitude of the hot-spot. As a next step, other models (e.g. Lagouarde and Irvine, 2008) will be tested, both in terms of capability to simulate the angular variability in the thermal infrared and in terms of possible operational implementation. 
Quality of estimated parameters of the kernel model depend on an adequate sampling of viewing and illumation angles. This is a general issue when calibrating geometric models based on information from geostationary and polar orbit satellites. In the present study, a representative sampling of illumination angles is achieved by calibrating the model using full-year time-series. In the case of the viewing angles, there are only limitations in azimuth angles (no observations from north) but these are expected to have small impact on the parameters because the solar kernel is symetric for relative azimuth angles.

Despite the limitations of the kernel model proposed by Vinikov et al (2012), the work presented here demonstrates that the developed methodology can be used to harmonize LST products for a reference view angle (e.g. nadir). In the framework of the LSA-SAF, this method will be used operationaly to generate an additional data layer to be disseminated within the LST product. This layer will consist of an estimate of the LST difference from the nadir to the SEVIRI view, thus providing the user with information on the expected deviation of the actual retrieval due to angular effects, on a pixel-by-pixel basis.

\section{ACKNOWLEDGMENTS}

This work was performed within the framework of ESA GlobTemperature project (http://www.globtemperature.info/). The SEVIRI/MSG LST data were generated by the LSA SAF project (http://landsaf.ipma.pt) funded by EUMETSAT. The MODIS LST data (MOD/MYD11) and Vegetation Continuous Fields data (MOD44B) were retrieved from the online Reverb/ECHO, courtesy of the NASA EOSDIS Land Processes Distributed Active Archive Center (LP DAAC), USGS/Earth Resources Observation and Science (EROS) Center, Sioux Falls, South Dakota (http://reverb.echo.nasa.gov). In situ observations made at Évora site were kindly provided by Dr Frank Göttsche from the Karlsruhe Institute of Technology (KIT). Research by Sofia L. Ermida was supported by the Portuguese Science Foundation (FCT) through the PhD grant SFRH/BD/96466/2013. 


\section{REFERENCES}

Barroso, C., Trigo, I. F., Olesen, F., DaCamara, C., \& Queluz,M. P. (2005). Intercalibration of NOAA and Meteosat window channel brightness temperatures. International Journal of Remote Sensing, 26(17), $3717-3733$

Belward, A. S. (1996). The IGBP-DIS global $1 \mathrm{~km}$ land cover data set (DISCover) - Proposal and implementation plans. IGBP-DIS working paper No. 13, IGBP-DIS Office, Météo-France, Toulouse, France (61 pp.).

Burrough, P. A., \& McDonell, R. A. (1998). Principles of Geographical Information Systems. Oxford University Press, New York, 190 pp.

Caselles, V., \& Sobrino, J. A. (1989). Determination of frosts in orange groves from NOAA-9 AVHRR data. Remote Sensing of Environment, 29 (1989), 135-146.

Duffour, C., A. Olioso, J. Demarty, C. Van der Tol, and J.-P. Lagouarde (2015): An evaluation of SCOPE: A tool to simulate the directional anisotropy of satellite-measured surface temperatures. Remote Sensing of Environment, 158, 362-375, doi:10.1016/j.rse.2014.10.019.

Ermida, S. L., Trigo, I. F., Dacamara, C. C., Göttsche, F. M., Olesen, F. S., \& Hulley, G. (2014). Validation of remotely sensed surface temperature over an oak woodland landscape - The problem of viewing and illumination geometries. Remote Sensing of Environment, 148, 16-27. 
Freitas, S. C., Trigo, I. F., Bioucas-dias, J. M., \& Göttsche, F. (2010). Quantifying the Uncertainty of Land Surface Temperature Retrievals From SEVIRI / Meteosat. IEEE Transactions on Geoscience and Remote Sensing, 48(1), 523-534.

García-Santos, V., Valor, E., Caselles, V., Ángeles Burgos, M., \& Coll, C. (2012). On the angular variation of thermal infrared emissivity of inorganic soils. Journal of Geophysical Research, 117, D19116. doi:10.1029/2012JD017931.

Göttsche, F.M., Olesen, F. S., \& Bork-Unkelbach, A. (2013). Validation of land surface temperature derived from MSG/SEVIRI with in situ measurements at Gobabeb, Namibia. International Journal of Remote Sensing, 34(9-10), 3069-3083.

Göttsche, F.M., Olesen, F. S., Trigo, I. F., Bork-Unkelbach, A., Martin, M. A. (2016). Long Term Validation of Land Surface Temperature Retrieved from MSG/SEVIRI with Continuous in-Situ Measurements in Africa. Remote Sensing, 8, 410.

Guillevic, P. C., Bork-Unkelbach, A., Göttsche, F. M., Hulley, G., Gastellu-Etchegorry, J. -P., Olesen, F. S., \& Privette, J. L. (2013). Directional viewing effects on satellite land surface temperature products over sparse vegetation canopies - A multisensor analysis. IEEE Geoscience and Remote Sensing Letters, 99, 1-5.

Jupp, D. L. B. (2000). A compendium of kernel \& other (semi-)empirical BRDF Models, report. Earth Observation Center, Office of Space Science Applicationsa, CSIRO, Clayton, Victoria, Australia. 
Labed, J., \& Stoll, M. P. (1991). Angular variation of land surface spectral emissivity in the thermal infrared: laboratory investigations on bare soils. International Journal of Remote Sensing, 12(11), 2299-2310. DOI: 10.1080/01431169108955259

Lagouarde, J.-P., Ballans, H., Moreau, P., Guyon, D., \& Coraboeuf, D. (2000). Experimental study of brightness surface temperature angular variations of maritime pine (Pinus pinaster) stands. Remote Sensing of Environment, 72(1), 17-34. doi:10.1016/S0034-4257(99)00085-1.

Lagouarde, J.-P., Moreau, P., Irvine, M., Bonnefond, J. M., Voogt, J. A., \& Solliec, F. (2004). Airborne experimental measurements of the angular variations in surface temperature over urban areas: Case study of Marseille (France). Remote Sensing of Environment, 93(4), 443-462. doi:10.1016/j.rse.2003.12.011.

Lagouarde, J.-P., \& Irvine, M. (2008). Directional anisotropy in thermal infrared measurements over Toulouse city centre during the CAPITOUL measurement campaigns: first results. Meteorology and Atmospheric Physics, 102, 173-185, doi: 10.1007/s00703-008-0325-4.

Li, Z.-L., Tang, B. H., Wu, H., Ren, H. Z., Yan, G. J., Wan, Z.M., Trigo, I. F., \& Sobrino, J. A. (2013). Satellite-derived land surface temperature: current status and perspectives. Remote Sensing of Environment, 131, 14-37. DOI:10.1016/j.rse.2012.12.008

Minnis, P., \& Khaiyer, M. M. (2000). Anisotropy of land surface temperature derived from satellite data. Journal of Applied Meteorology, 39(7), 1117-1129. 
Norman, J. M., \& Becker, F. (1995). Terminology in thermal infrared remote sensing of natural surfaces. Agricultural and Forest Meteorology, 77(3-4), 153-166. DOI:10.1016/0168-1923(95)02259Z

Peres, L. F., \& DaCamara, C. C. (2005). Emissivity maps to retrieve land-surface temperature from MSG/SEVIRI. IEEE Transactions on Geoscience and Remote Sensing, 43 (2005), 1834-1844.

Pinheiro, A. C. T., Privette, J. L., Mahoney, R., \& Tucker, C. J. (2004). Directional effects in a daily AVHRR land surface temperature dataset over Africa. IEEE Transactions on Geoscience and Remote Sensing, 42(9), 1941-1954. http://doi.org/10.1109/TGRS.2004.831886

Pinheiro, A. C. T., Privette, J. P., \& Guillevic, P. C. (2006). Modeling the observed angular anisotropy of land surface temperature in a Savanna. IEEE Transactions on Geoscience and Remote Sensing, 44(4), 1036-10467.

Rasmussen, M. O., Pinheiro, A. C., Proud, S. R, \& Sandholt, I. (2010) Modeling angular dependance in Land Surface Temperatures from SEVIRI instrument onboard the geostationary Meteosat Second Generation. IEEE Transactions on Geoscience and Remote Sensing, 48, 3123-3133. doi: 10.1109/TGRS.2010.2044509

Rasmussen, M. O., Göttsche, F., Olesen, F., \& Sandholt, I. (2011). Directional Effects on Land Surface Temperature Estimation From Meteosat Second Generation for Savanna Landscapes. IEEE Transactions on Geoscience and Remote Sensing, 49(11), 4458-4468. 
Ren, H., Yan, G.,Chen, L., \& Li Z. (2011). Angular effect of MODIS emissivity products and its application to the split-window algorithm. ISPRS Journal of Photogrammetry and Remote Sensing, 66, 498-507. doi: 10.1016/j.isprsjprs.2011.02.008.

Salomonson, V. V., Barnes, W., \& Masuoka, E. J. (2006). Introduction to MODIS and an overview of associated activities. Earth Science Satellite Remote Sensing, 1, 12-32.

Seemann, S. W., Borbas, E. E., Knuteson, R. O., Stephenson, G. R., \& Huang, H.-L. (2007). Development of a Global Infrared Land Surface Emissivity Database for Application to Clear Sky Sounding Retrievals from Multi-spectral Satellite Radiance Measurements. Journal of Applied Meteorology and Climatology, 47, 108-123.

Sobrino, J. A., \& Cuenca, J. (1999). Angular variation of thermal infrared emissivity for some natural surfaces from experimental measurements. Applied Optics, 38, 3931-3936.

Townshend, J.R.G., Carroll, M., Dimiceli, C., Sohlberg, R., Hansen, M., \& DeFries, R. (2011). Vegetation Continuous Fields MOD44B, 2001 Percent Tree Cover, Collection 5, University of Maryland, College Park, Maryland, 2001.

Trigo, I. F., Monteiro, I. T., Olesen, F., \& Kabsch, E. (2008). An assessment of remotely sensed land surface temperature. Journal of Geophysical Research, 113(D17), 1-12. http://doi.org/10.1029/2008JD010035

Trigo, I. F., DaCamara, C. C., Viterbo, P., Roujean, J. L., Olesen, F., Barroso, C., et al. (2011). The satellite application facility on land surface analysis. International Journal of Remote Sensing, 32(10), $2725-2744$. 
Trigo, R.M., Añel, J., Barriopedro, D., García-Herrera, R., Gimeno, L., Nieto, R., Castillo, R., Allen, M.R., \& Massey, N. (2013). The record Winter drought of 2011-12 in the Iberian Peninsula, in Explaining Extreme Events of 2012 from a Climate Perspective. Bulletin of the American Meteorological Society, 94 (9), S41-S45

Vinnikov, K. Y., Yu, Y., Goldberg, M. D., Tarpley, D., Romanov, P., Laszlo, I., \& Chen, M. (2012). Angular anisotropy of satellite observations of land surface temperature. Geophysical Research Letters, 39(23), L23802. doi:10.1029/2012GL054059

Wan, Z. M., \& Dozier, J. (1996). A generalized split-window algorithm for retrieving land- surface emissivity from space. IEEE Transactions on Geoscience and Remote Sensing, 34, 892-905.

Wan, Z.M., Zhang, Y. L., Zhang, Q. C., \&Li, Z. L. (2002). Validation of the land-surface temperature products retrieved from Terra Moderate Resolution Imaging Spectro radiometer data. Remote Sensing of Environment, 83(2), 163-180

Wan, Z., \& Li, Z. -L. (2008). Radiance-based validation of the V5 MODIS land-surface temperature product. International Journal of Remote Sensing, 29(17-18), 5373-5395. http://doi.org/10.1080/01431160802036565

Wan, Z. (2014). New refinements and validation of the collection-6 MODIS land-surface temperature/emissivity product. Remote Sensing of Environment. 140, 36-45.

Wang, A., Barlage, M., Zeng, X., \& Draper, C. S. (2014). Comparison of land skin temperature from a land model, remote sensing, and in situ measurement. Journal of Geophysical Research: Atmospheres, 119, 3093-3106, doi:10.1002/2013JD021026. 
Xu, T., Liu, S., Liang, S., \& Qin, J. (2011). Improving predictions of water and heat fluxes by assimilating MODIS. Journal of Hydrometeorology, 12, 227-244. 